\title{
A Novel Memcapacitor Model and Its Application for Generating Chaos
}

\author{
Guangyi Wang, ${ }^{1}$ Shiyi Jiang, ${ }^{1}$ Xiaowei Wang, ${ }^{2}$ Yiran Shen, ${ }^{1}$ and Fang Yuan ${ }^{1}$ \\ ${ }^{1}$ Institute of Modern Circuits and Intelligent Information, Hangzhou Dianzi University, Hangzhou 310018, China \\ ${ }^{2}$ Department of Automation, Shanghai University, Shanghai 200072, China \\ Correspondence should be addressed to Xiaowei Wang; laura423_wang@163.com
}

Received 30 August 2016; Revised 13 November 2016; Accepted 20 November 2016

Academic Editor: Mingshu Peng

Copyright (c) 2016 Guangyi Wang et al. This is an open access article distributed under the Creative Commons Attribution License, which permits unrestricted use, distribution, and reproduction in any medium, provided the original work is properly cited.

\begin{abstract}
Memristor and memcapacitor are new nonlinear devices with memory. We present a novel memcapacitor model that has the capability of capturing the behavior of a memcapacitor. Based on this model we also design a chaotic oscillator circuit that contains a HP memristor and the memcapacitor model for generating good pseudorandom sequences. Its dynamic behaviors, including equilibrium points, stability, and bifurcation characteristics, are analyzed in detail. It is found that the proposed oscillator can exhibit some complex phenomena, such as chaos, hyperchaos, coexisting attractors, abrupt chaos, and some novel bifurcations. Moreover, a scheme for digitally realizing this oscillator is provided by using the digital signal processor (DSP) technology. Then the random characteristics of the chaotic binary sequences generated from the oscillator are tested via the test suit of National Institute of Standards and Technology (NIST). The tested randomness definitely reaches the standards of NIST and is better than that of the well-known Lorenz system.
\end{abstract}

\section{Introduction}

In 1971, Chua deduced the existence of memristor by the completeness of basic circuit elements [1]. In 2008, Strukov et al. from HP labs successfully realized a memristor using $\mathrm{TiO}_{2}$ material and nanotechnology [2]. Memristor and its applications in nonlinear circuits have attracted immense worldwide interest from both academia and industry.

Memcapacitor is member of a large family of new circuit elements postulated by Chua in the late seventies and presented as one of 4 guest lectures at the 1978 European Conference on Circuit Theory and Design (ECCTD) [3]. Memcapacitor is formally defined by Chua in 2003 [4]. More recently, memcapacitor and meminductor were presented at the keynote lecture of the First Symposium on Memristors, held in Berkeley, California, in 2008 [5]. The nanoscale circuit elements, that is, memristor, memcapacitor, and meminductor, have memorial properties and can store information without power supplies. Hence, memorial nanodevices became a hot point of research in electronics and material science.
With the realization of nanoscale memristor, the memristor-based chaotic circuit is widely studied. In the proposed memristor-based oscillators, at present, most models of memristors are piecewise linear or quadratic or cubic smooth functions $[6,7]$. Based on the piecewise linear model, a simplest chaotic circuit was presented, which has only three elements in series: a linear capacitor, a linear inductor, and a memristor [8]. In [9], a chaotic circuit based on the realistic model of the HP memristor is introduced. The circuit makes use of two HP memristors in antiparallel.

On the contrary to memristor, there are fewer research reports on the circuit that contains memcapacitor or the combination of memcapacitor and memristor.

Although actual solid-state memcapacitor has not been yet realized so far, it is important to design effective memcapacitor models and make prospective studies for its applications [10]. In 2009, a piecewise linear memcapacitor model was first presented in [5]. Then a memcapacitor-based chaotic oscillator was constructed by using the memcapacitor model with the piecewise linear $\varphi-\sigma$ characteristic [11]. Some other memcapacitor models were established. Reference 


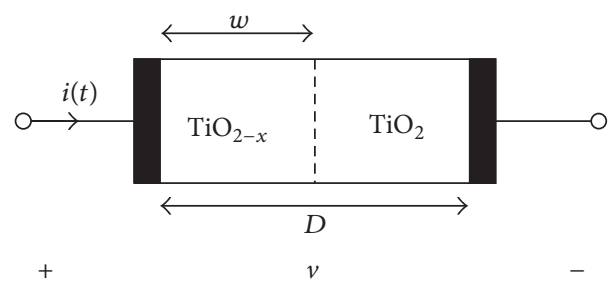

FIgURE 1: Physical model of HP memristor.

[12] presented a mutator for transforming memristor into memcapacitor, and [13]designed a memcapacitor emulator based on a memristor. For convenience [10] described a floating memcapacitor emulator that can be practically applied in electronic circuits. For simplicity, a new memcapacitor emulator without using any memristor was proposed in [14], and different behavioral memcapacitor models [15-17] were successively proposed.

Meanwhile, in order to explore the circuit characteristics of memristor, memcapacitor, and meminductor, some chaotic oscillators based on these memory elements were designed [18-22], and some special phenomena were found, such as coexistence attractors, hidden attractors, and extreme multistability.

Reference [23] introduced an emulator of floating memcapacitor and meminductor by using current conveyors. Reference [24] describes a novel 5-component chaotic circuit based on a charge-controlled memcapacitor; it provides a thorough theoretical analysis of the circuit and extensive simulation results accurately.

The above-mentioned chaotic oscillators contain mostly only one memory device. So we knew little about the properties of the circuit that contains multimemory devices. And in general, the practical application circuits of the memory devices are the hybrid circuits of memristor, memcapacitor, and meminductor. Aiming at this problem, this paper proposes a charge-controlled memcapacitor model and designs a chaotic circuit using a HP memristor and the memcapacitor model for exploring the characteristics of nonlinear circuits containing memristor and memcapacitor. We analyze the complex nonlinear dynamic behaviors of this circuit and find some important dynamical properties. In addition, a DSP experiment is designed for confirming the proposed oscillator. Finally, its random characteristics are tested by the NIST standards.

\section{2. $\mathrm{HP} \mathrm{TiO}_{2}$ Memristor Model}

In 2008, a physical device with memristor function was realized by nanolevel $\mathrm{TiO}_{2}$ thin film in $\mathrm{HP}$ labs. The physical model is shown in Figure 1.

One of the layers is missing some of the oxygen atoms, which is called doping layer $\left(\mathrm{TiO}_{2-x}\right.$ layer $)$ and has stronger electrical conductivity. The other layer is a pure $\mathrm{TiO}_{2}$ layer without impurities, which is called a nondoped layer with high resistance. The expression for the $\mathrm{TiO}_{2}$ memristor is

$$
\begin{aligned}
M & =R_{\text {on }} \frac{w}{D}+R_{\text {off }}\left(1-\frac{w}{D}\right), \\
\frac{d w}{d t} & =\mu_{v} \frac{R_{\text {on }}}{D} i(t),
\end{aligned}
$$

where $M$ is called memristance; $R_{\text {on }}$ and $R_{\text {off }}$ are the low resistance and high resistance for $w=D$ and $w=0$, respectively; $\mu_{v}$ is the dopant mobility; $i(t)$ is the current through the memristor; $w$ is the thickness of $\mathrm{TiO}_{2-x}$ layer and $D$ represents the total thickness of the memristor.

By Ohm's law, we have

$$
v(t)=M i(t)=\left(R_{\mathrm{on}} \frac{w(t)}{D}+R_{\mathrm{off}}\left(1-\frac{w(t)}{D}\right)\right) i(t) .
$$

Inserting (1) into (2), we can simplify it to

$$
\begin{aligned}
v(t) & =\left(R_{\mathrm{off}}-\frac{R_{\mathrm{off}}-R_{\mathrm{on}}}{D^{2}} \mu_{v} R_{\mathrm{on}} \int_{-\infty}^{t} i(\tau) d \tau\right) i(t) \\
& =\left(a-b \int_{-\infty}^{t} i(\tau) d \tau\right) i(t) \\
& =R\left(\int_{-\infty}^{t} i(\tau) d \tau\right) i(t),
\end{aligned}
$$

where $a=R_{\text {off }}, b=\left(R_{\text {off }}-R_{\text {on }}\right) \mu_{v} R_{\text {on }} / D^{2}$.

\section{Memcapacitor Model Based on $\mathrm{HP} \mathrm{TiO}_{2}$ Memristor}

In 2009, Di Ventra et al. extended the concept of memory devices from memristor to memcapacitor and meminductor, where the memcapacitor is defined as [5]

$$
\begin{aligned}
q(t) & =C_{M}(x, v, t) v(t), \\
\dot{x} & =f(x, v, t), \\
v(t) & =C_{M}^{-1}(x, q, t) q(t), \\
\dot{x} & =f(x, q, t),
\end{aligned}
$$

where $q(t)$ is the quantity of the charge at time $t, v(t)$ is the corresponding voltage across the memcapacitor, $x$ is an internal variable of the memcapacitor, and $C_{M}$ and $C_{M}^{-1}$ are the memcapacitance and the inverse memcapacitance, respectively. The above definition of memcapacitor can be simplified to [5]

$$
\begin{aligned}
& q(t)=C_{M}\left[\int_{t_{0}}^{t} v(\tau) d \tau\right] v(t)=C_{M}[\varphi] v(t), \\
& v(t)=C_{M}^{-1}\left[\int_{t_{0}}^{t} q(\tau) d \tau\right] q(t)=C_{M}^{-1}[\sigma] q(t)
\end{aligned}
$$

called voltage-controlled and charge-controlled memcapacitors, respectively. 


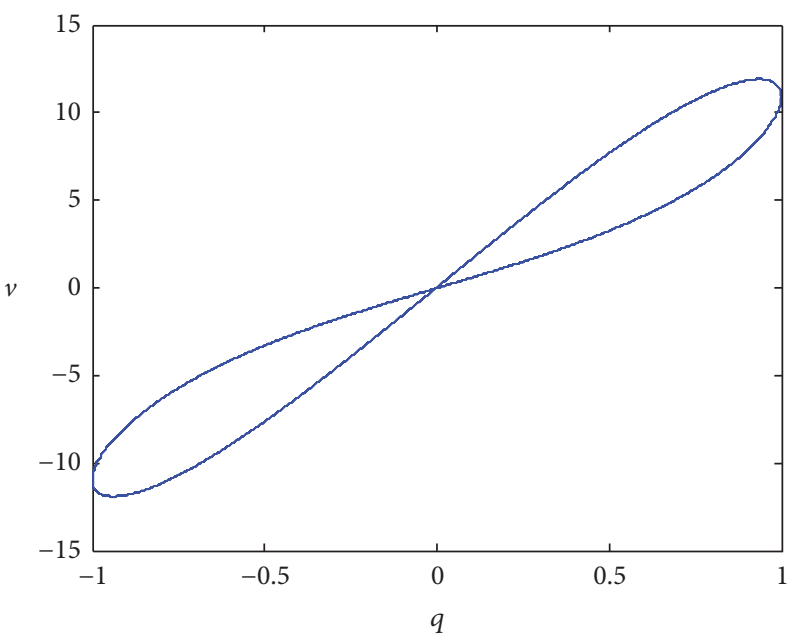

(a)

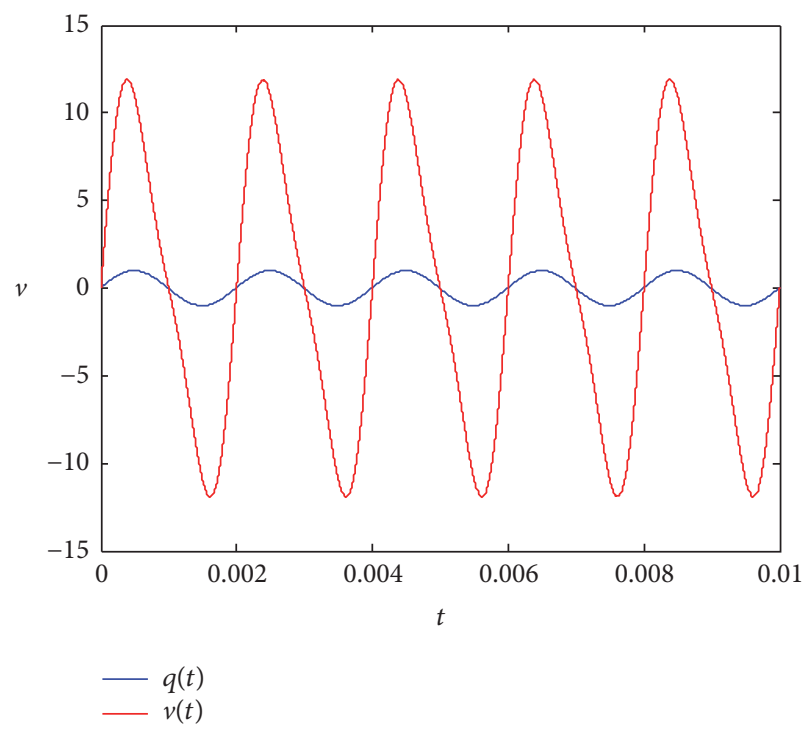

(b)

FIGURE 2: Hysteretic curve and waveform of the memcapacitor. (a) Voltage-charge hysteretic curve. (b) Waveforms of voltage and charge.

Nanoscale devices such as memristor, memcapacitor, and meminductor are common at the nanoscale, where the dynamical characteristics of ions and electrons are likely to depend on the history of the system that exhibit their memorability [5]. From the points of mathematics and circuit, we infer that memcapacitor and memristor have the same constitutive relation for the $\varphi-\sigma$ of memcapacitor and $\varphi-q$ of memristor, respectively.

Analogous to (3), we obtain the simplified model of a memcapacitor as

$$
q(t)=\left(a-b \int_{t_{0}}^{t} v(\tau) d \tau\right) v(t)
$$

where $a-b \int_{t_{0}}^{t} v(\tau) d \tau$ is the memcapacitance $C_{M}[\varphi]$.

Inverse memcapacitance and memcapacitance follow the same law except that they are reciprocal. So we can write down the mathematical expression of inverse memcapacitance according to (6):

$$
v(t)=\left(c-d \int_{t_{0}}^{t} q(\tau) d \tau\right) q(t)
$$

where $\left(c-d \int_{t_{0}}^{t} q(\tau) d \tau\right)$ is the inverse memcapacitance $C_{M}^{-1}[\varphi]$.

An emulator of the memcapacitor, that is, (6) or (7), can be realized by an equivalent analog electronic circuit, which can implement the operations of (6) or (7).

In the practical circuit applications, common is the charge-controlled memcapacitor. So we choose the expression described in (7) as the model of memcapacitor. The physical parameters of $\mathrm{TiO}_{2}$ memristor, where $R_{\text {on }}=100 \Omega$, $R_{\text {off }}=16000 \Omega, \mu_{v}=10^{-10} \mathrm{~cm}^{2} \mathrm{~s}^{-1} \mathrm{~V}^{-1}$, and $D=10 \mathrm{~nm}$, are equivalent to set $c=16, d=15900$ in memcapacitor model. Assume that the input of the memcapacitor is $q(t)=\sin (1000 \pi t)$; then the voltage-charge curve and their

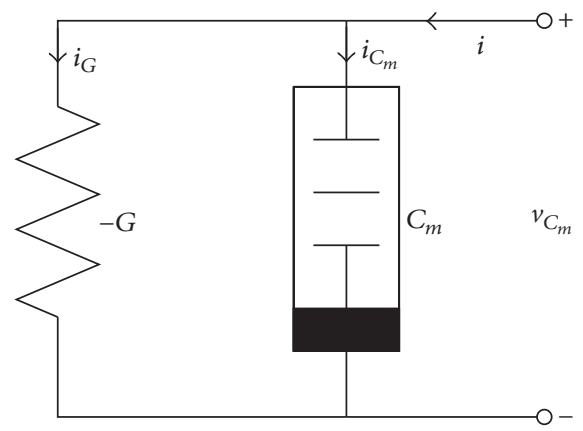

FIgURE 3: Parallel connection of a memcapacitor and a negative conductor.

corresponding waveforms of the memcapacitor are shown in Figure 2.

When a memcapacitor and a negative conductor are in parallel, shown in Figure 3, the parallel circuit can be equivalent to an active capacitive circuit that can provide energy to maintain oscillation for a memcapacitor-based oscillator. The total current and voltage of the parallel circuit are $i=i_{C_{m}}+i_{G}$ and $v=v_{C_{m}}=v_{G}$, respectively, and then the total charge of the parallel circuit can be described as

$$
q=\int_{t_{0}}^{t} i d t=\int_{t_{0}}^{t}\left(i_{C_{m}}+i_{G}\right) d t=q_{C_{m}}+q_{G}
$$

where $q_{C_{m}}$ denotes the charge of memcapacitor $C_{m}, q_{G}$ is the charge of negative resistor, and

$$
\begin{aligned}
q_{G} & =\int-G v_{C_{m}}(\tau) d \tau=-G \int\left(c-d \sigma_{C_{m}}\right) q_{C_{m}} d \tau \\
& =-G\left(c \sigma_{C_{m}}-0.5 d \sigma_{C_{m}}^{2}\right) .
\end{aligned}
$$




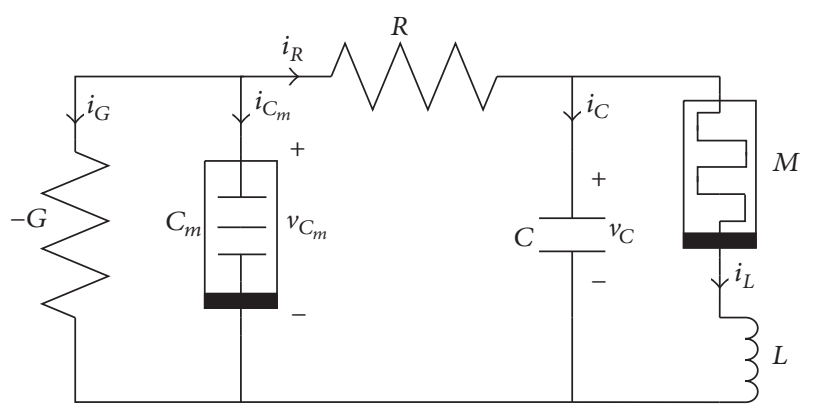

Figure 4: Nonlinear circuit containing a memristor and a memcapacitor.

So the parallel circuit can be equivalent to an active capacitive circuit, in which its voltage $v_{C_{m}}$ and charge $q$ are

$$
\begin{aligned}
v_{C_{m}} & =\left(c-d \sigma_{C_{m}}\right) q_{C_{m}}, \\
q & =q_{C_{m}}+q_{G}, \\
& =q_{C_{m}}-G\left(c \sigma_{C_{m}}-0.5 d \sigma_{C_{m}}^{2}\right),
\end{aligned}
$$

where $\sigma_{C_{m}}=\int q_{C_{m}} d t$, called integration variable of charge in this paper.

\section{Chaotic Circuit Based on Memcapacitor and Memristor}

We design a nonlinear circuit, as shown in Figure 4, which contains an active memcapacitor $C_{m}^{\prime}$ (a memcapacitor in parallel with a negative resistor), a $\mathrm{TiO}_{2}$ memristor $M$, a linear inductor $L$, a linear capacitor $C$, and a linear resistor $R$.

The state equations of the proposed circuit can be obtained by Kirchhoff's current and voltage laws:

$$
\begin{aligned}
C \frac{d v_{C}}{d t} & =-i_{L}+\frac{1}{R}\left(v_{C_{m}}-v_{C}\right), \\
L \frac{d i_{L}}{d t} & =v_{C}-M i_{L}, \\
\frac{d q_{C_{m}}}{d t} & =G v_{C_{m}}+\frac{1}{R}\left(v_{C}-v_{C_{m}}\right), \\
\frac{d q_{M}}{d t} & =i_{L},
\end{aligned}
$$

where $v_{C_{m}}=\left(c-d \sigma_{C_{m}}\right) q_{C_{m}}, M=a-b q_{M}$, and $\sigma_{C_{m}}=\int q_{C_{m}} d t$. If we let $x=v_{C}, y=i_{L}, z=q_{C_{m}}, w=\sigma_{C_{m}}, v=q_{M}, m=1 / L$, $n=1 / C, j=1 / R$, and $k=G$, (11) can be simplified to the following equations:

$$
\begin{aligned}
& \frac{d x}{d t}=n(j((c-d w) z-x)-y), \\
& \frac{d y}{d t}=m(x-(a-b v) y), \\
& \frac{d z}{d t}=k(c-d w) z+j(x-(c-d w) z), \\
& \frac{d w}{d t}=z, \\
& \frac{d v}{d t}=y,
\end{aligned}
$$

where the fourth equation of (12) is an added equation according to the relation $\sigma_{C_{m}}=\int q_{C_{m}} d t$.

Let the circuit parameters $m=7.35, n=0.17, j=4.8$, and $k=2.1$, the memristor parameters $a=0.01$ and $b=0.1$, and the memcapacitor parameters $c=0.7$ and $d=-2.0$. When the initial value is $(0.01,0.10,0.01,0.01,0.01)$, system (12) possesses a chaotic attractor with the Lyapunov exponents: $L_{1}=0.0836, L_{2}=0.0065, L_{3}=0, L_{4}=-0.0080$, and $L_{5}=-2.0474$. The projections of the $5 \mathrm{D}$ chaotic attractor on the $2 \mathrm{D}$ plane are shown in Figure 5. The projection of Poincaré mapping trajectory on $z=0$ cross section is shown in Figure 6(a); and the continuous waveforms of the partial state variables $x, z$, and $v$ are shown in Figure 6(b).

\section{Basic Dynamic Behaviors}

5.1. Equilibrium Point Set and Stability. Let $\dot{x}=\dot{y}=\dot{z}=\dot{w}=$ $\dot{v}=0$; the equilibrium points of (12) can be obtained as a set:

$$
E=\left\{(x, y, z, w, v) \mid x=y=z=0, w=c_{1}, v=c_{2}\right\} .
$$

Thus every point in $w-v$ plane is the equilibrium point of the system, where $c_{1}$ and $c_{2}$ are arbitrary real numbers, implying that the system has an infinite number of equilibria. The Jacobi matrix of the system at the equilibrium set is

$$
\text { Jacobi }=\left[\begin{array}{ccccc}
-j n & -n & j n C_{m} & 0 & 0 \\
m & -m M & 0 & 0 & 0 \\
j & 0 & r C_{m} & 0 & 0 \\
0 & 0 & 1 & 0 & 0 \\
0 & 1 & 0 & 0 & 0
\end{array}\right] \text {, }
$$

where $C_{m}=c-d c_{1}, M=a-b c_{2}$, and $r=k-j$. Taking circuit parameters as $m=7.35, n=0.17, j=4.8, k=2.1, a=0.01$, $b=0.1, c=0.7$, and $d=-2.0$, we get the characteristic equation of equilibrium point set as

$$
\lambda^{3}+p \lambda^{2}+q \lambda^{1}+l=0
$$

where

$$
\begin{aligned}
& p=j n-r C_{m}+m M, \\
& q=n\left(m-j C_{m}(j-r)+j m M\right)-m r M C_{m}, \\
& l=-n(m r+j M(m r+j)) C_{m} .
\end{aligned}
$$




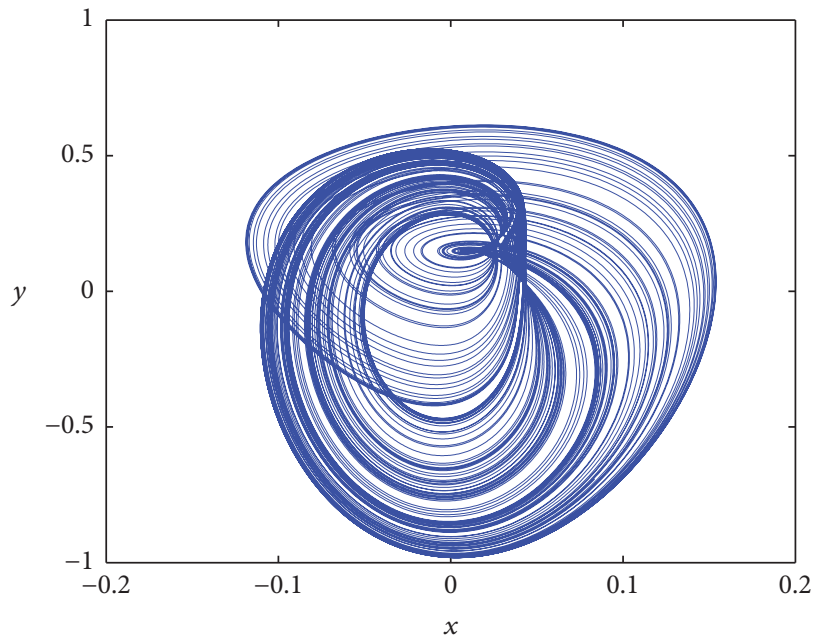

(a)

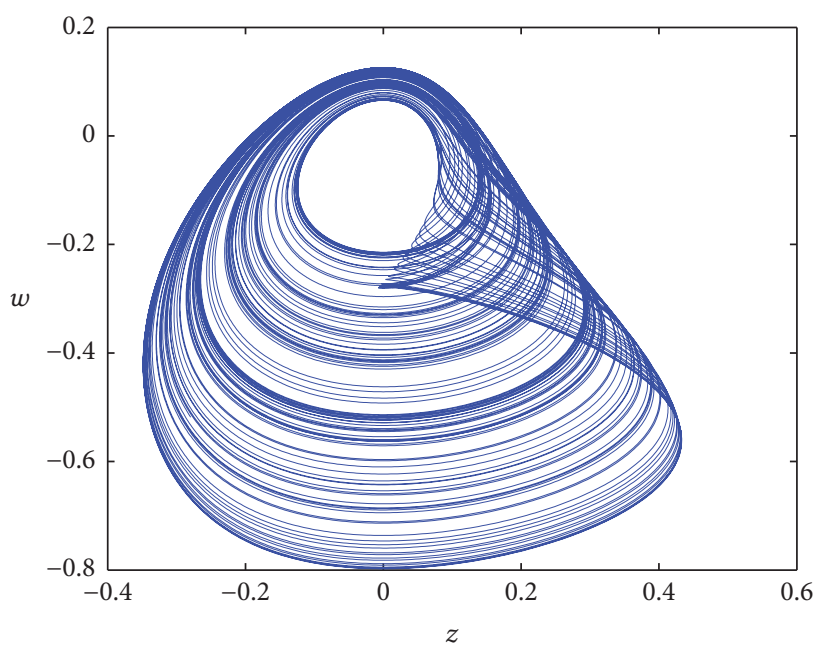

(c)

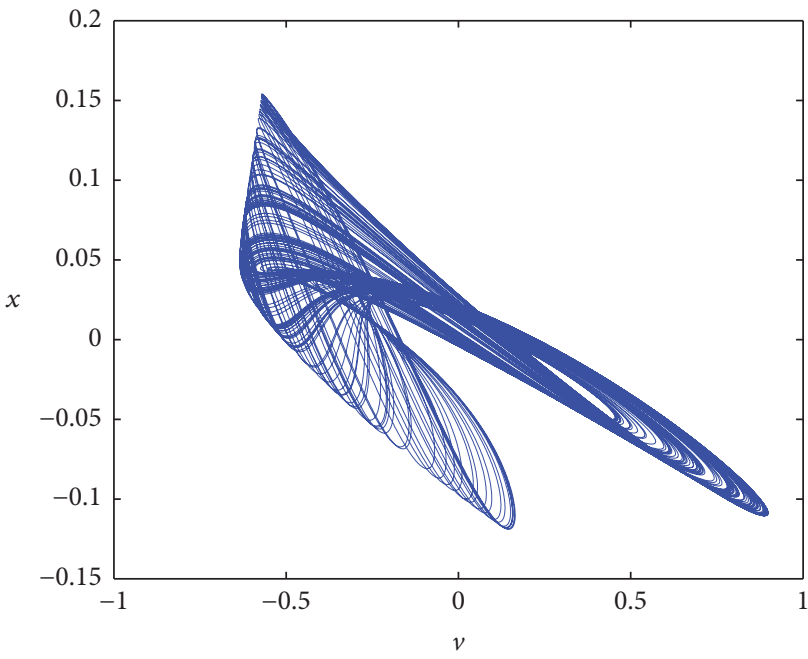

(e)

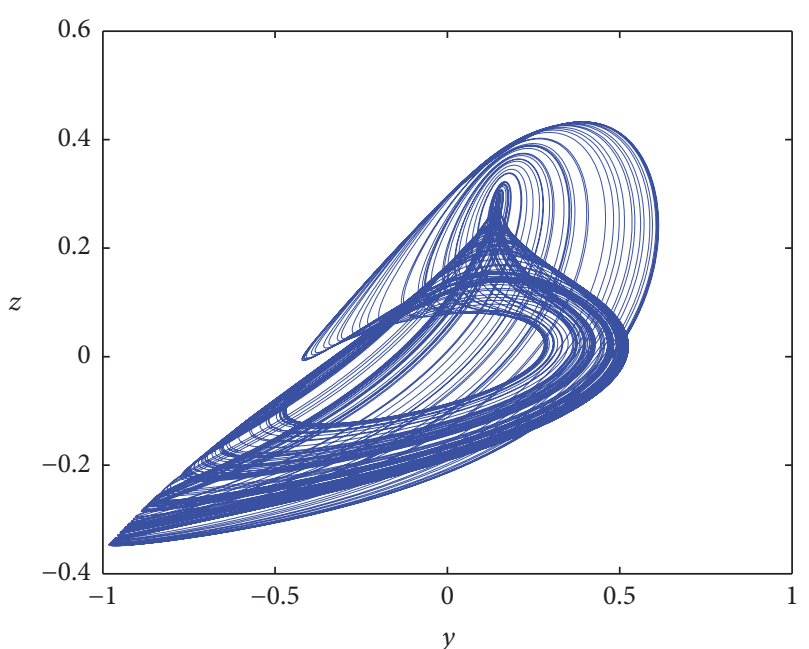

(b)

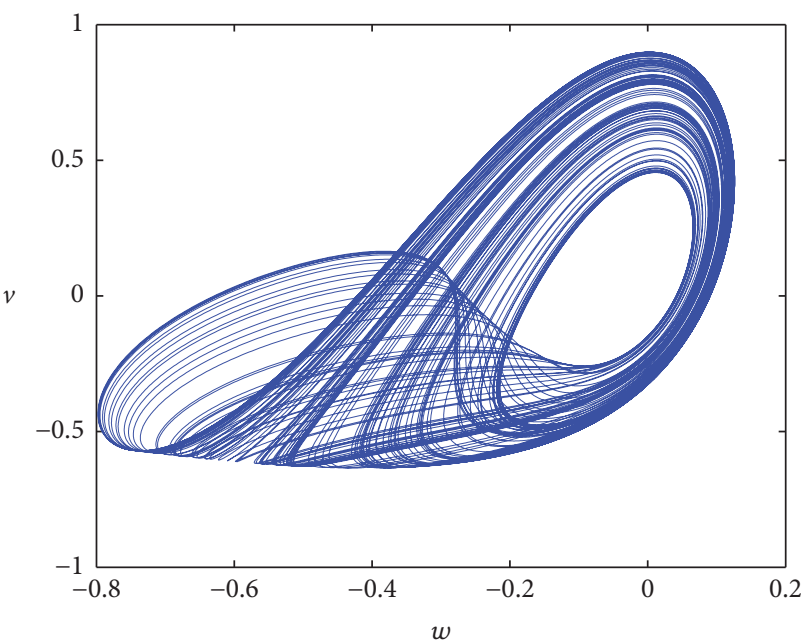

(d)

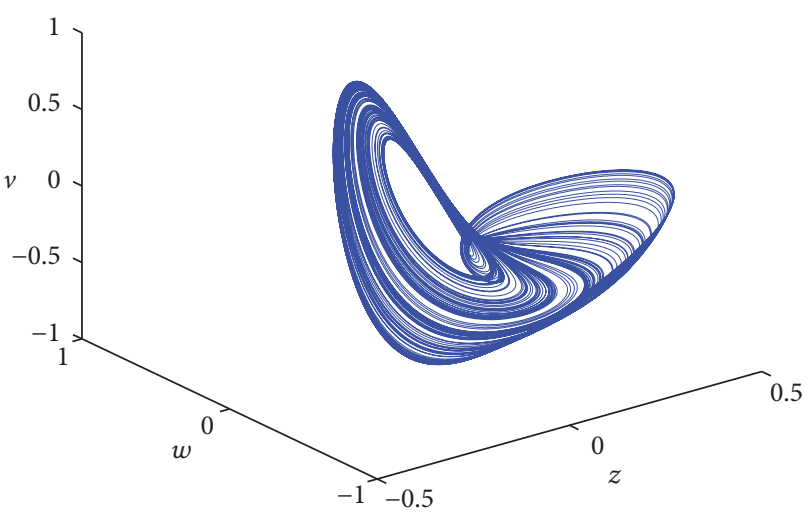

(f)

Figure 5: Chaotic attractors of the oscillator. (a) $x-y$ phase plane. (b) $y-z$ phase plane. (c) $z-w$ phase plane. (d) $w-v$ phase plane. (e) $v-x$ phase plane. (f) $z-w-v$ phase space. 


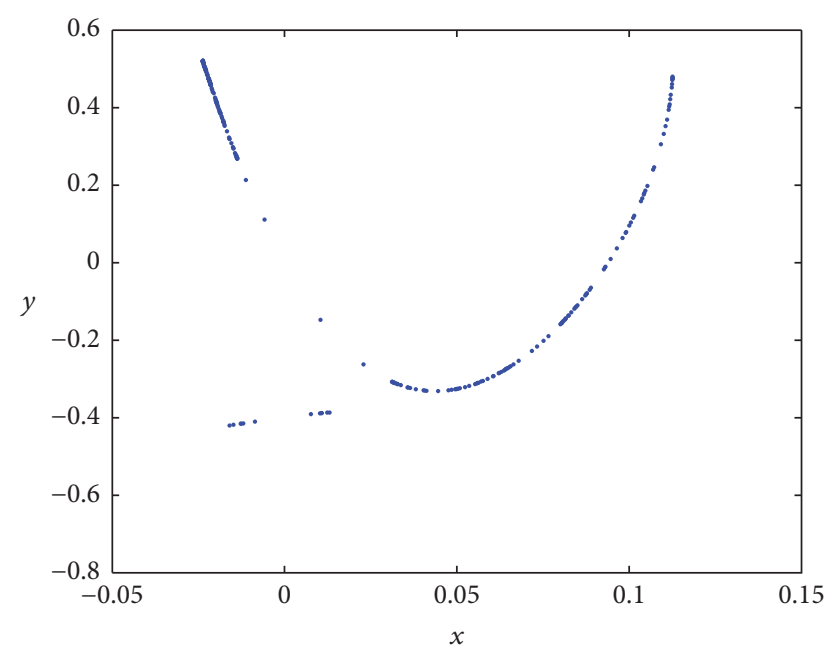

(a)
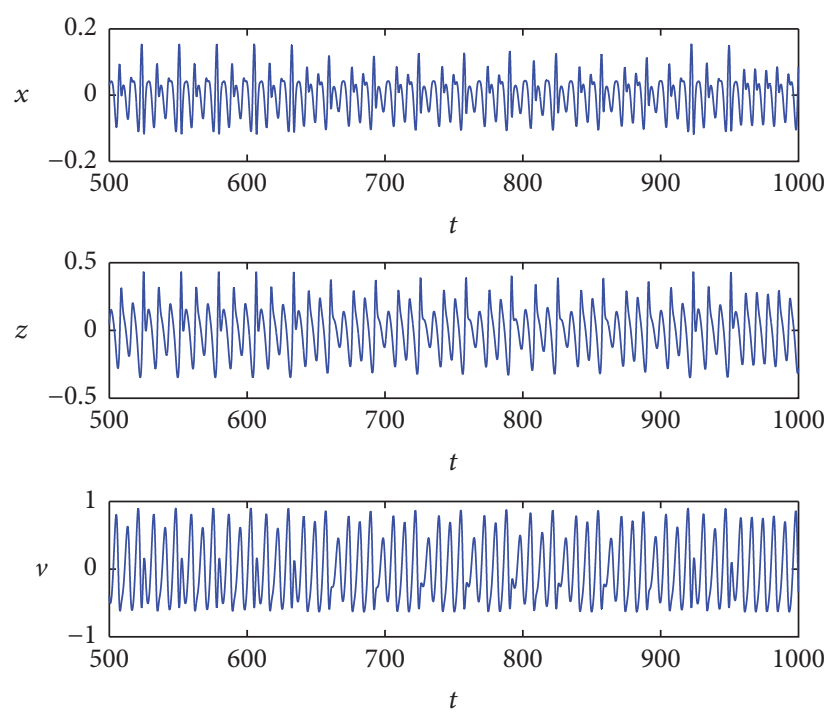

(b)

Figure 6: (a) Projection of Poincaré mapping. (b) Continuous waveforms of state variables $x, z$, and $v$.

Equation (15) demonstrates that (12) has two zero characteristic roots and three nonzero characteristic roots, and the coefficients of $\lambda$ are all nonzero real numbers. By Routh-Hurwitz stability conditions, we can get the coefficient determinant:

$$
\Delta_{5}=\left|\begin{array}{lllll}
a_{1} & a_{0} & 0 & 0 & 0 \\
a_{3} & a_{2} & a_{1} & a_{0} & 0 \\
a_{5} & a_{4} & a_{3} & a_{2} & a_{1} \\
a_{7} & a_{6} & a_{5} & a_{4} & a_{3} \\
a_{9} & a_{8} & a_{7} & a_{6} & a_{5}
\end{array}\right|=\left|\begin{array}{lllll}
p & 1 & 0 & 0 & 0 \\
l & q & p & 1 & 0 \\
0 & 0 & l & q & p \\
0 & 0 & 0 & 0 & l \\
0 & 0 & 0 & 0 & 0
\end{array}\right| .
$$

If $a_{0}>0$, the necessary and sufficient condition for the stability of the system is that principal minors of each order are greater than zero; thus

$$
\Delta_{1}, \Delta_{2}, \Delta_{3}, \Delta_{4}, \Delta_{5}>0
$$

Now the real parts of the three nonzero characteristic roots are all negative, implying that the system is stable. If principal minors of each order are not all positive, that is, at least one is less than or equal to zero, the system is unstable. When circuit parameters are $m=7.35, n=0.17, j=4.8$, $k=2.1, a=0.01, b=0.1, c=0.7, d=-2.0, c_{1}=0.1, c_{2}=0.1$, and $a_{0}=1$, the principal minors of each order are

$$
\begin{aligned}
& \Delta_{1}=p=3.246 \\
& \Delta_{2}=p q-l=-11.2126, \\
& \Delta_{3}=l p q-l^{2}=-34.0449, \\
& \Delta_{4}=0 \\
& \Delta_{5}=0
\end{aligned}
$$

So, the system is unstable, which fulfills the condition to produce chaos.

5.2. Influence of Parameter $b$ on Dynamic Characteristics. When $a=0.01, c=0.7, d=-0.8, m=7.35, n=0.17, j=4.8$, and $k=2.1$, the bifurcation diagram and Lyapunov exponent spectrum of the system along with the change of parameter $b$ are shown in Figure 7. For clarity, the fifth Lyapunov exponent curve is not drawn in Figure 7(b). In this paper, the maximum value method is used to draw the bifurcation diagram and the Jacobi method is used to calculate the Lyapunov exponents.

From Figure 7(a), it can be seen that system (12) enters chaotic state via an irregular period bifurcation and a perioddoubling bifurcation and then evolves eventually into a periodic orbit via an inverse period-doubling bifurcation process.

Obviously, there are many periodic windows in chaotic regions of the system. For example, when $b$ is in the range $[-0.5,-0.028]$, the system is in the irregular periodic orbit while when $b$ is in $[-0.028,-0.016]$, the system is in perioddoubling orbit; when $b$ is in $[0.016,0.021]$ and $[0.09,0.13]$, the system enters the period-doubling orbit and inverse perioddoubling orbits from chaotic states, respectively. Figure 8 shows several periodic and chaotic orbits.

\subsection{Influence of Parameter $d$ on Dynamic Characteristics.} When parameter $d$ of the memcapacitor changes, using the Matlab, the corresponding bifurcation diagram and Lyapunov exponent spectrum about the state variable $x$ are shown in Figure 9, where the parameter $b=0.01$ and other parameters remain unchanged.

With the varying of $d$, the system undergoes a complex bifurcation process. When $d$ is in $[-5.5,-2.52]$, the system is in periodic orbit. Then the system enters chaotic state from the periodic orbit in the region $[-2.52,1.05]$, in 


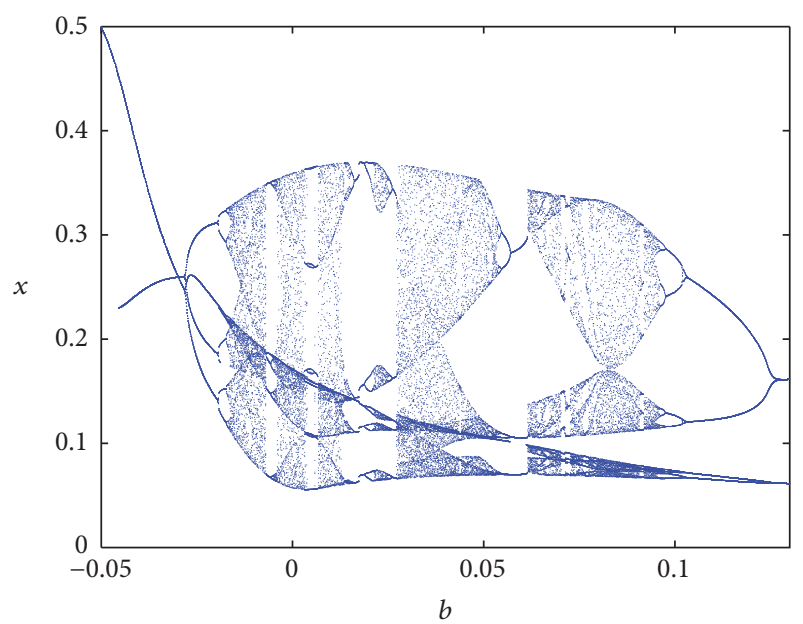

(a)

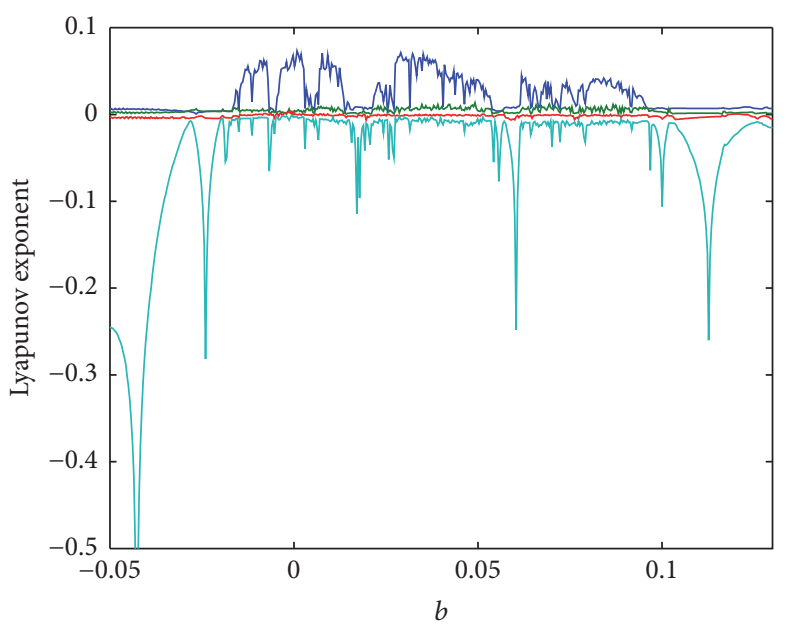

(b)

FIgURE 7: Bifurcation and Lyapunov exponents of $x$ versus $b$. (a) Bifurcation diagram. (b) Lyapunov exponent spectrum.

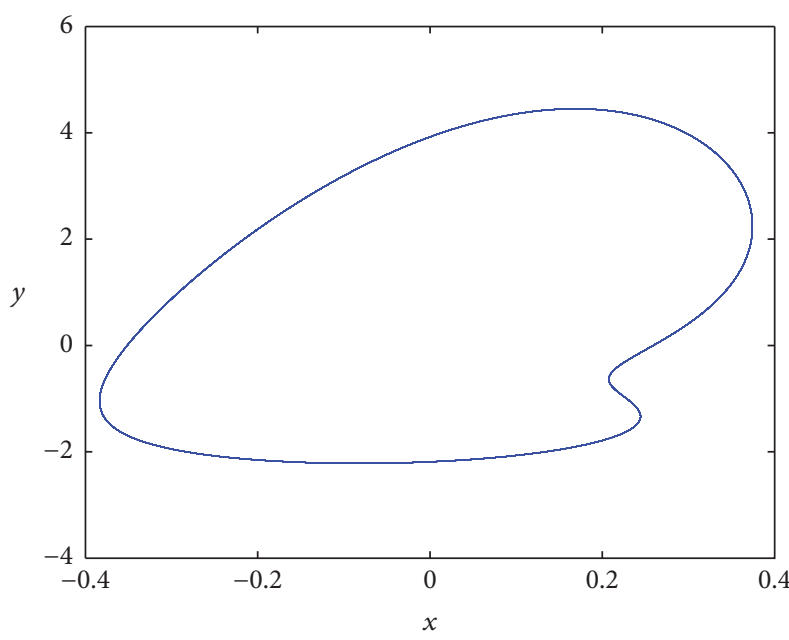

(a)

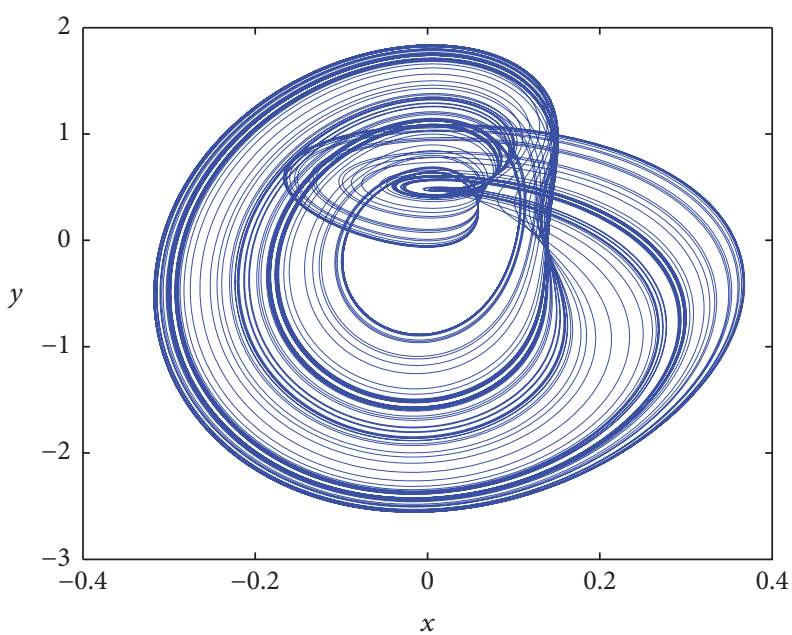

(c)

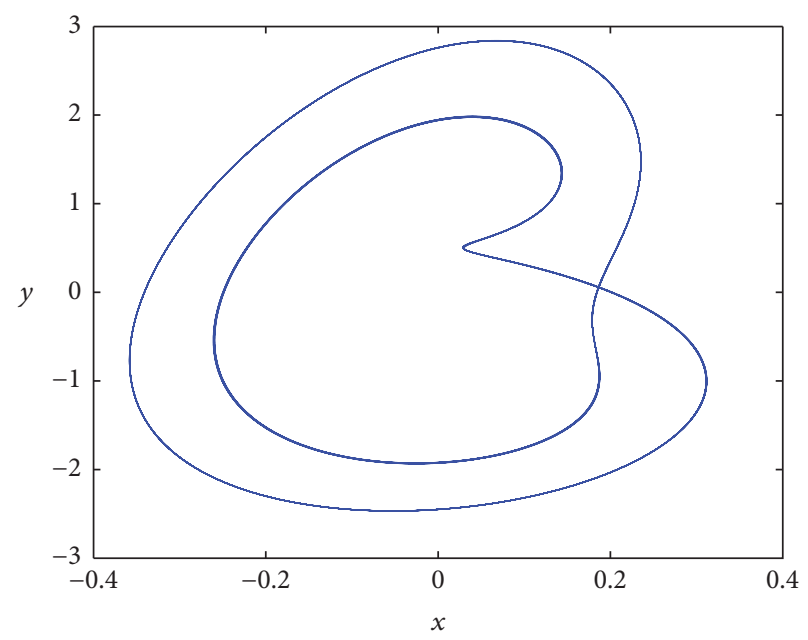

(b)

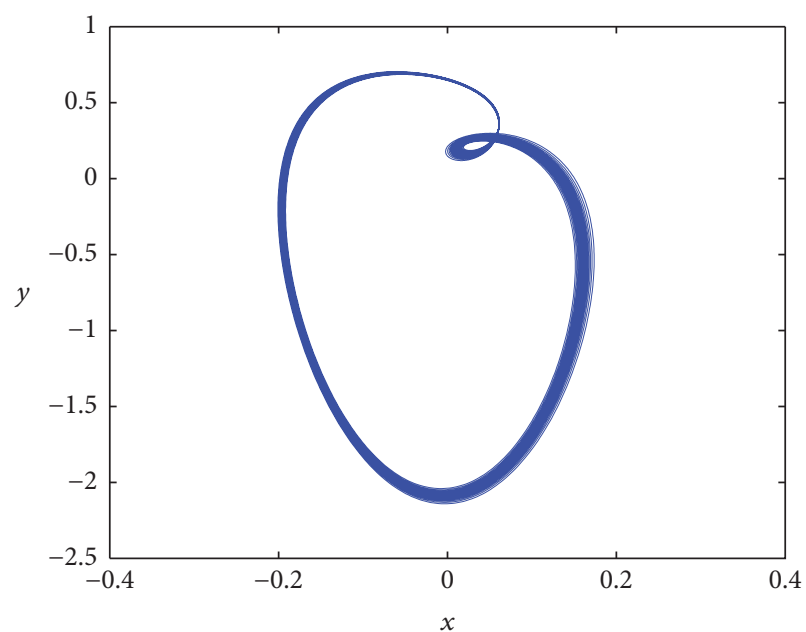

(d)

Figure 8: Chaotic phase diagrams on $x-y$ plane versus $b$. (a) $b=-0.04$. (b) $b=-0.02$. (c) $b=0.01$. (d) $b=0.13$. 


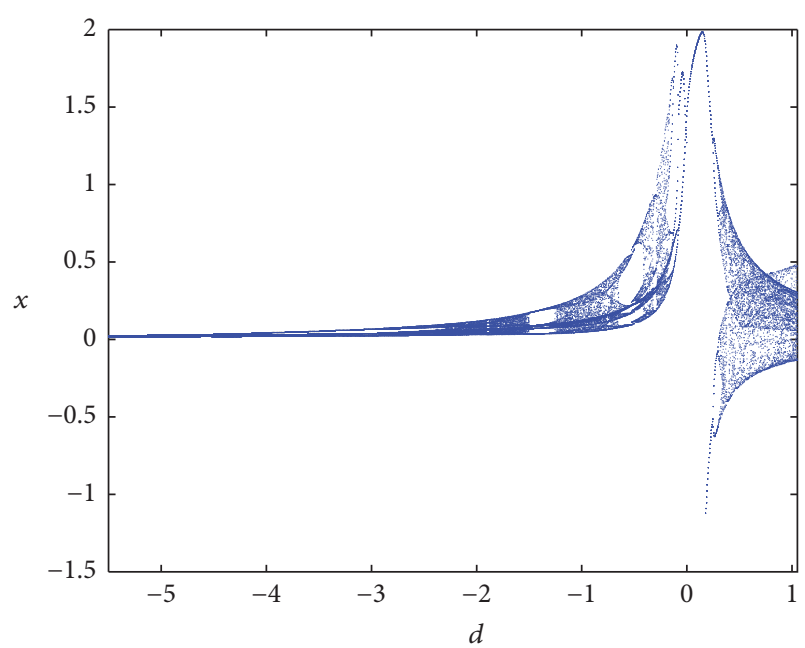

(a)

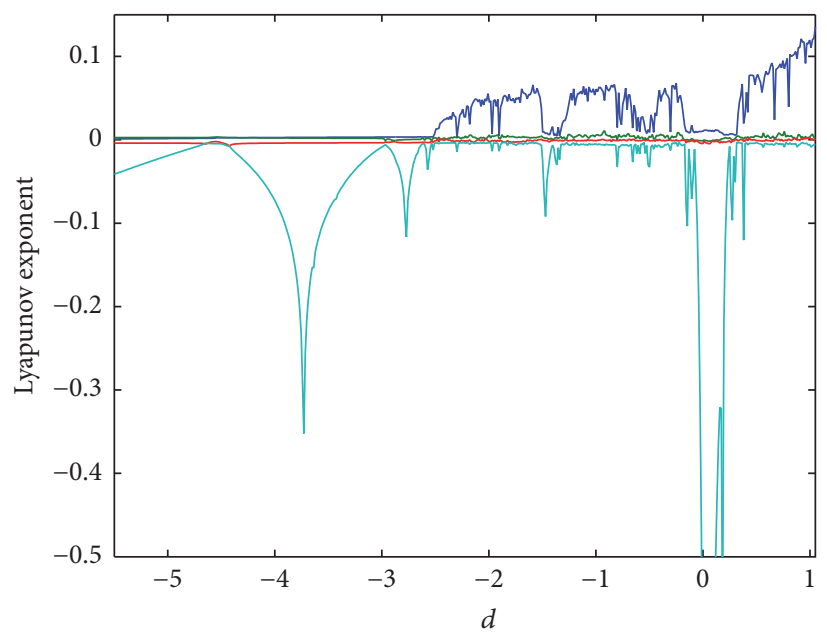

(b)

FiguRE 9: Bifurcation and Lyapunov exponents versus $d$. (a) Bifurcation diagram. (b) Lyapunov exponents.

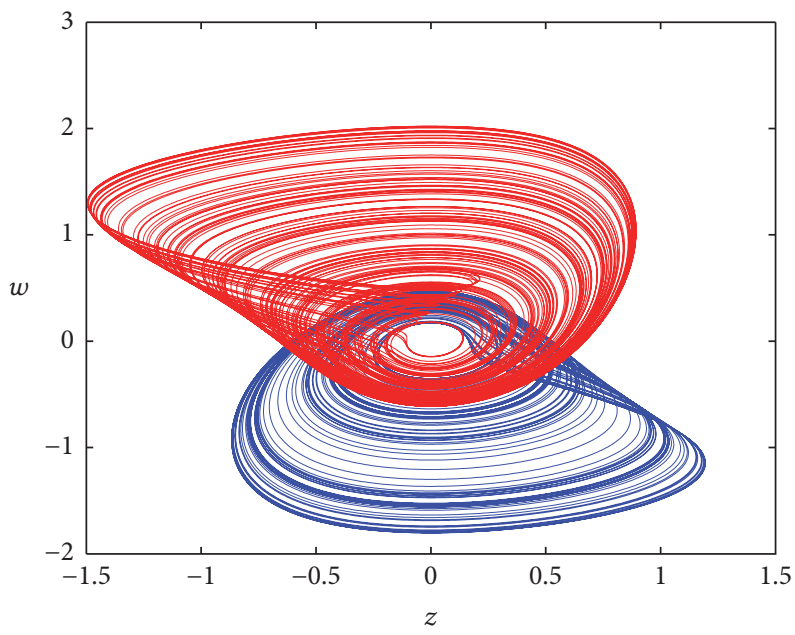

(a)

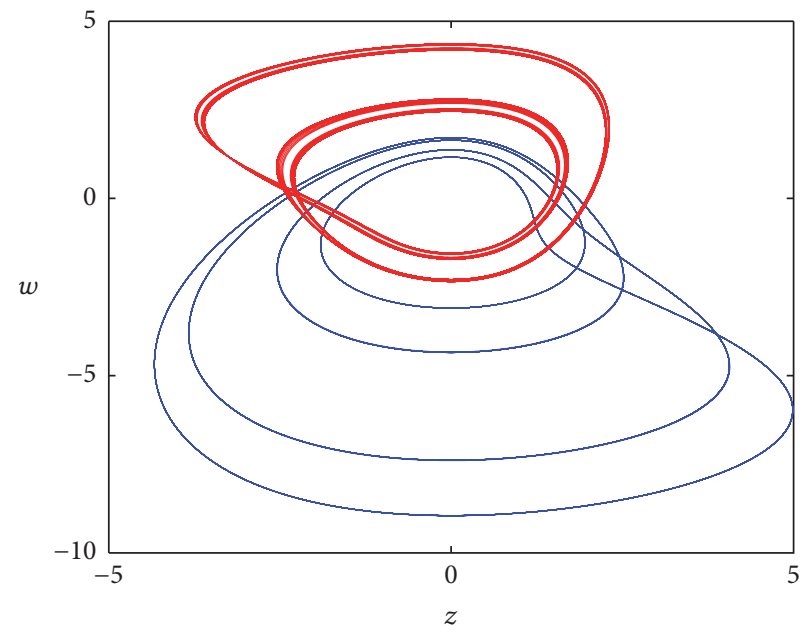

(b)

Figure 10: Phase diagrams on $z$-w plane versus $d$. (a) Chaotic attractors with $d=-0.8$ (blue) and $d=0.8$ (red). (b) Limit cycles with $d=-0.15$ (blue) and $d=0.15$ (red).

which the system exhibits three obvious periodic windows: $[-1.496,-1.326],[-0.617,-0.4595]$, and $[-0.1575,0.3149]$.

It is different from a general memristor-based system on the two side regions of the third periodic window, where the phase diagram of the running trajectory changes abruptly, as illustrated in Figure 10. When $d<0$, the chaotic attractor and limit cycle lie under part of the phase diagrams and are depicted as blue. When $d>0$, the chaotic attractor and limit cycle lie in the upper part of the phase diagrams and are depicted as red.

5.4. Influence of Parameters $m$ and $n$ on Dynamic Characteristics. When other parameters remain unchanged and if the parameter $m$ is changed within the range $[6.95,8.15]$, the bifurcation diagram and Lyapunov exponent spectrum of state variable $x$ are shown in Figure 11, where the parameter $m$ is the reciprocal of the inductance $L$. The routes leading to chaos are generally from period or quasi-period bifurcations. However, the circuit has no solutions as $m<6.95$, but at $m=6.95$ it suddenly turns into chaos without bifurcations, which is called abrupt chaos. In this case, we observe weak hyperchaos near the parameter region $m=7.1$, which has two positive Lyapunov exponents. Bifurcation and Lyapunov exponent spectrum of state variable $x$ versus parameter $n$ are similar to those versus parameter $m$, as shown in Figure 12, in which we also observe weak hyperchaos near the parameter region $m \in[0.12,0.18]$.

Furthermore, if other parameters are fixed and the parameters $m$ and $n$ are changed simultaneously, the $2 \mathrm{D}$ bifurcation, that is, the dynamic map, can be given as in Figure 13, which shows the Lyapunov exponents of the chaotic system versus two parameters. In the dynamical map, the 


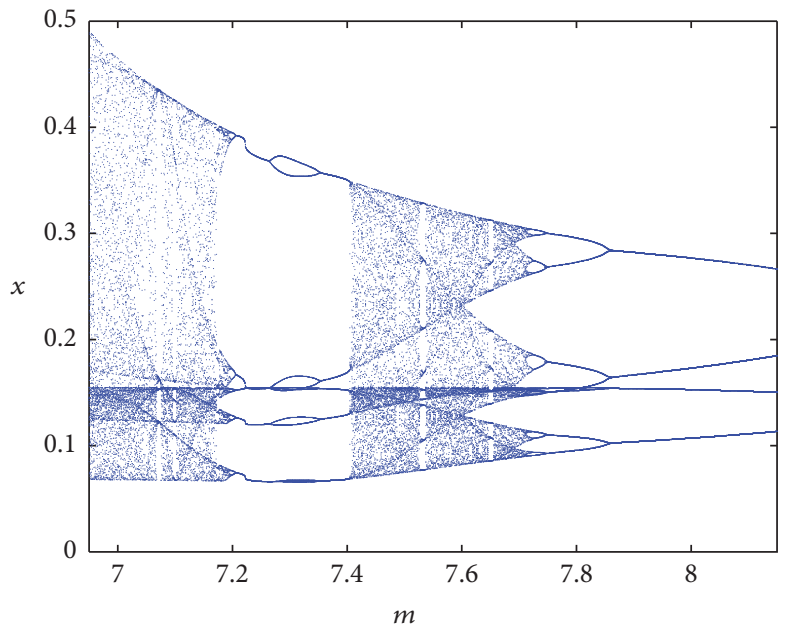

(a)

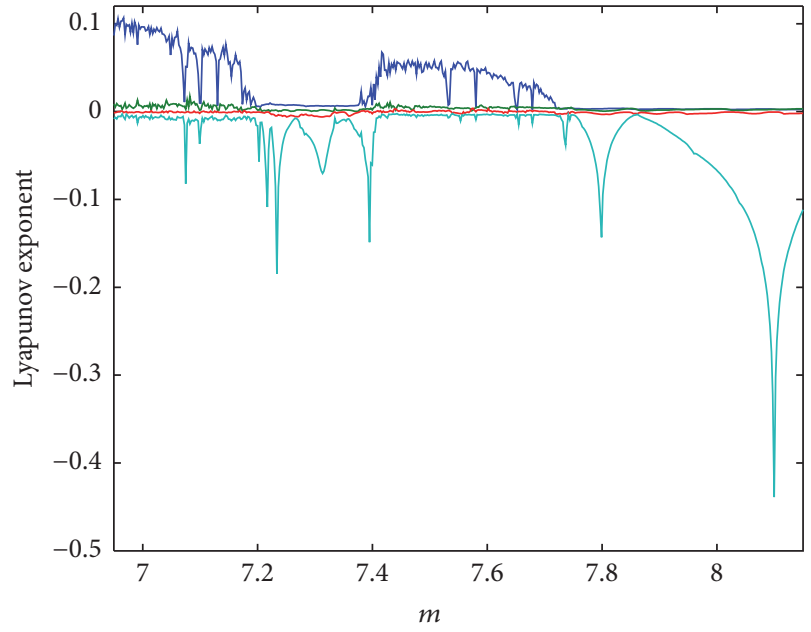

(b)

FIGURE 11: Bifurcation and Lyapunov exponents versus parameter $m$. (a) Bifurcation diagram. (b) Lyapunov exponent spectrum.

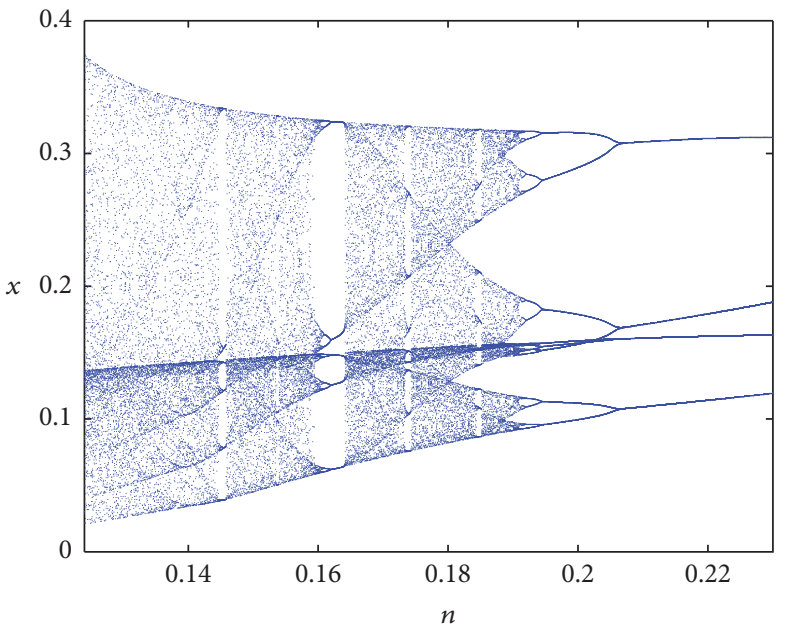

(a)

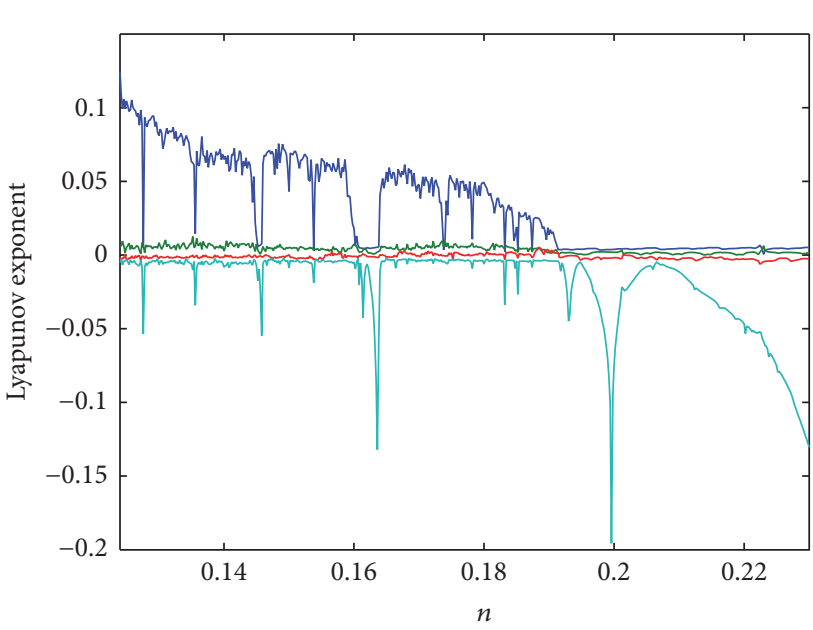

(b)

FIGURE 12: Bifurcation diagram (a) and Lyapunov exponent spectrum (b) versus the change of parameter $n$.

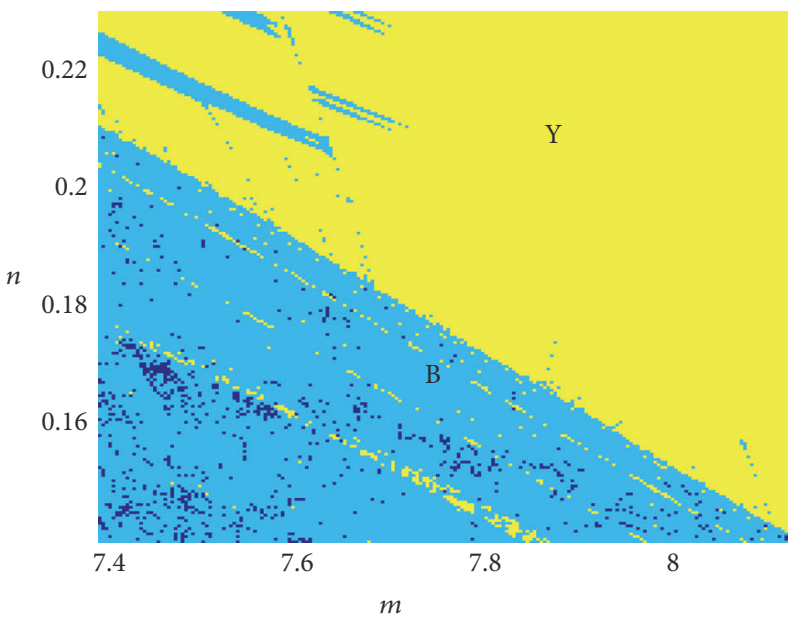

FIGURE 13: Dynamic map (the Lyapunov exponent versus parameters $m$ and $n$ simultaneously at the same time). 


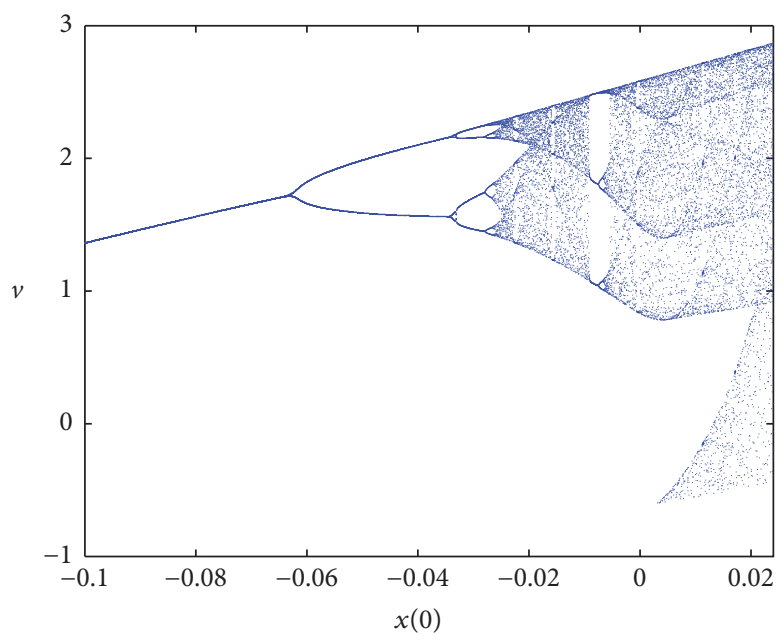

(a)

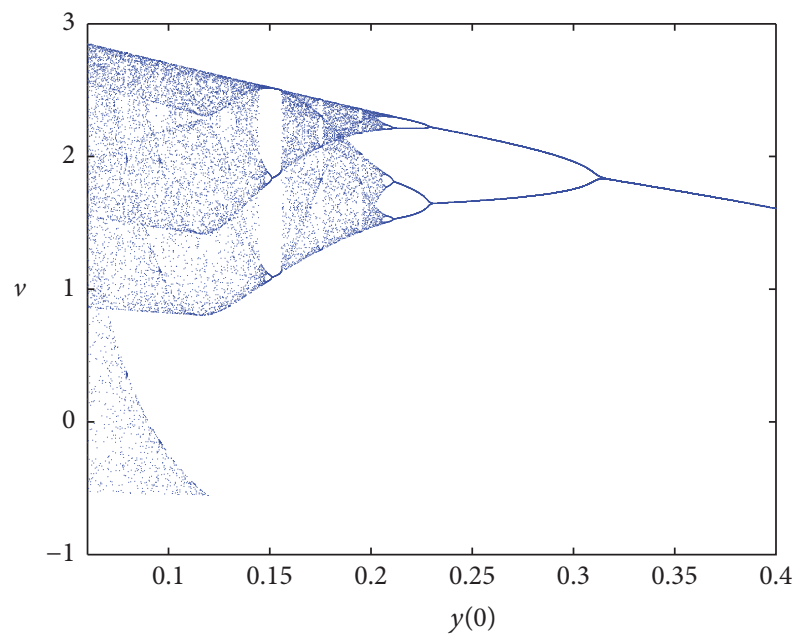

(c)

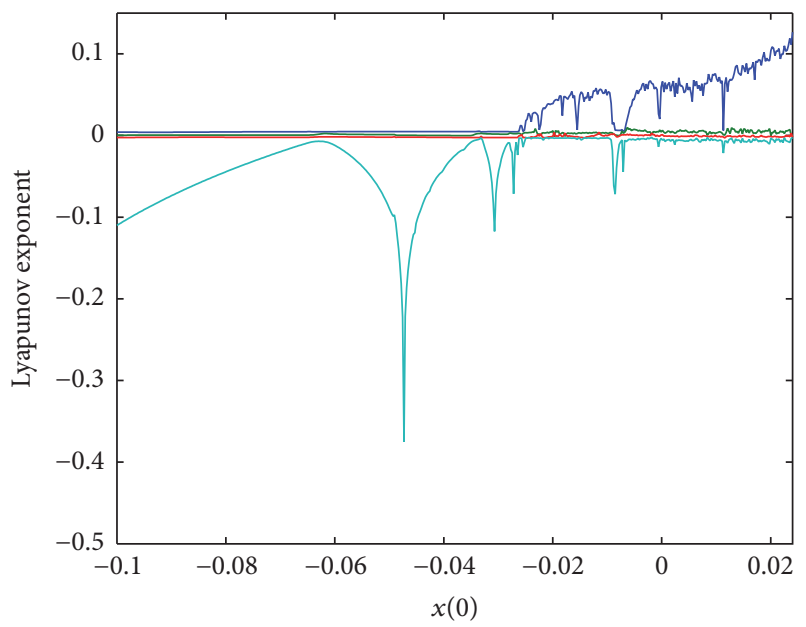

(b)

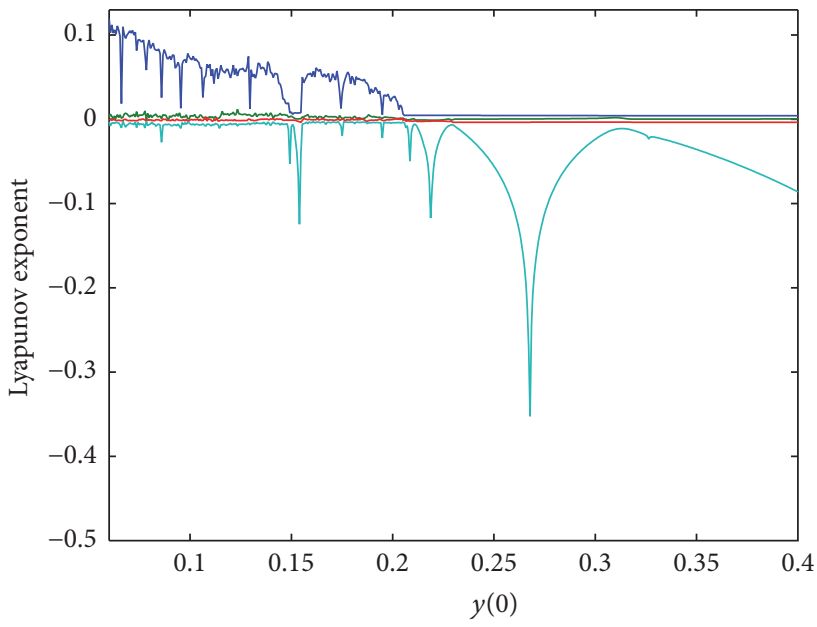

(d)

FiguRE 14: Bifurcation and Lyapunov exponents with initial values. (a) Bifurcation diagram versus $x(0)$. (b) Lyapunov exponents versus $x(0)$. (c) Bifurcation diagram versus $y(0)$. (d) Lyapunov exponents versus $y(0)$.

yellow parts labeled by $\mathrm{Y}$ represent the periodic domains, while the light blue parts and the dark blue parts which are labeled by B represent chaotic and hyperchaotic domains, respectively.

The blue region B, which is interspersed with some finely banded yellow areas, shows that there are multiple periodic windows in the chaotic domain. In addition, there are some irregularly distributed dark blue spots in the blue region, indicating there are some hyperchaotic states in the chaotic domain. On the other hand, there is a dividing line between the yellow and the blue in the middle of the map. The dividing line is a straight line with a negative slope, and the bottom left of the line is a chaotic region while the upper right part is a periodic orbit region. It indicates that the system will be gradually evolved into periodic orbit at the boundary from chaotic orbit. Coexistent Oscillation. In general, chaotic orbit has high sensitivity to initial values but chaotic state is irrelevant to the initial values in its attractive basin. Furthermore, periodic orbit and periodic state are all not sensitive to system initial conditions. However, system (12) can enter different states in different initial conditions; that is, the state of this system has high sensitive to its initial values, not only for chaotic state but also for periodic state, which is called coexistent oscillation.

Figure 14 shows the bifurcation diagram and the Lyapunov exponent spectrum of the system with the changes of initial conditions $x(0)$ and $y(0)$. The variation ranges of $x(0)$ and $y(0)$ are $[-0.1,0.024]$ and $[0.06,0.4]$, and the other initial conditions are all 0.01 , where the system parameters are $a=0.01, b=0.01, c=0.7, d=-0.8, m=7.35, n=0.16$, $j=4.8$, and $k=2.1$.

From Figure 15, we can see when $x(0)$ changes, the evolution of the state of system (12) is opposite to that of $y(0)$. With the increase of $x(0)$, the system enters chaotic orbit via a period-doubling orbit and evolves eventually a chaotic 


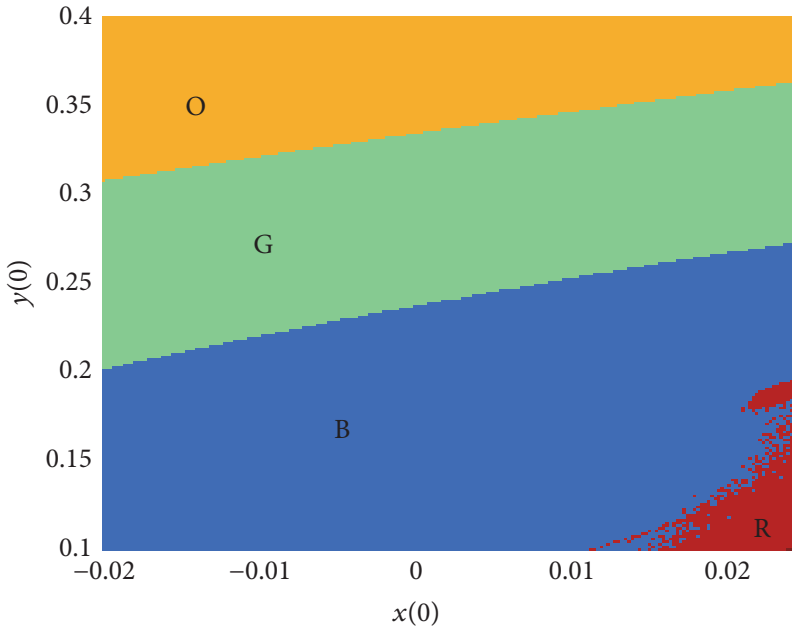

(a)

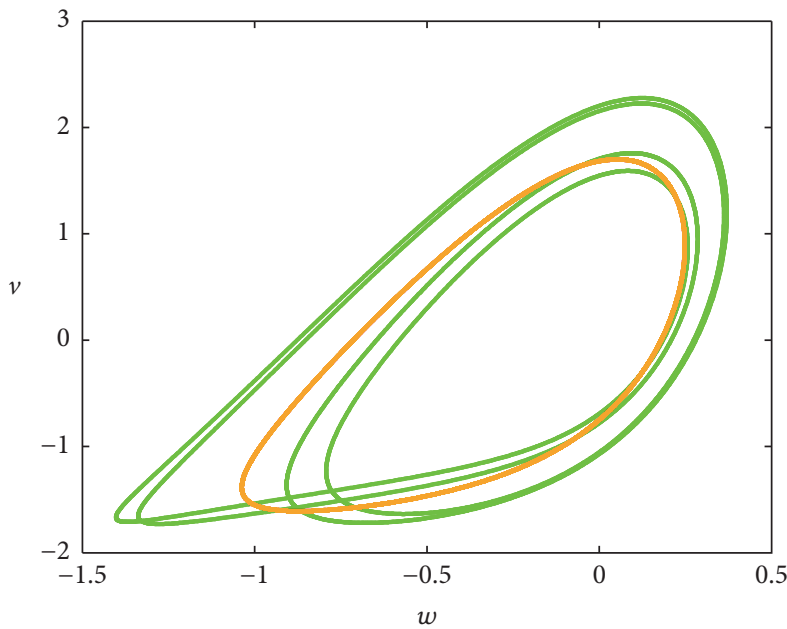

(c)

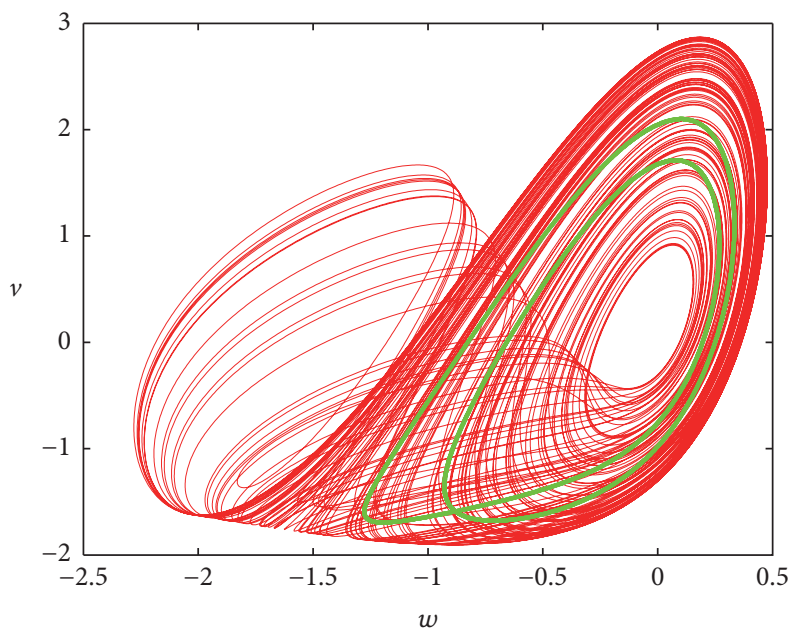

(e)

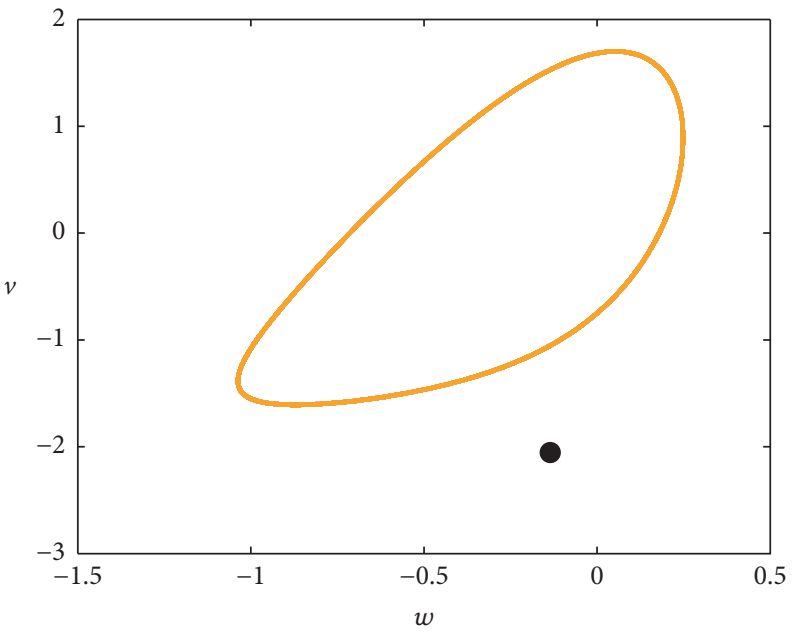

(b)

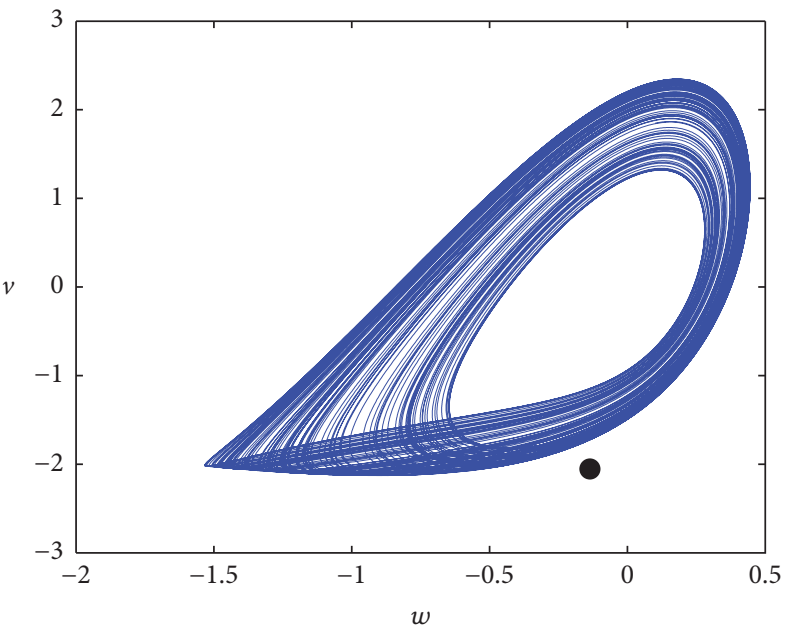

(d)

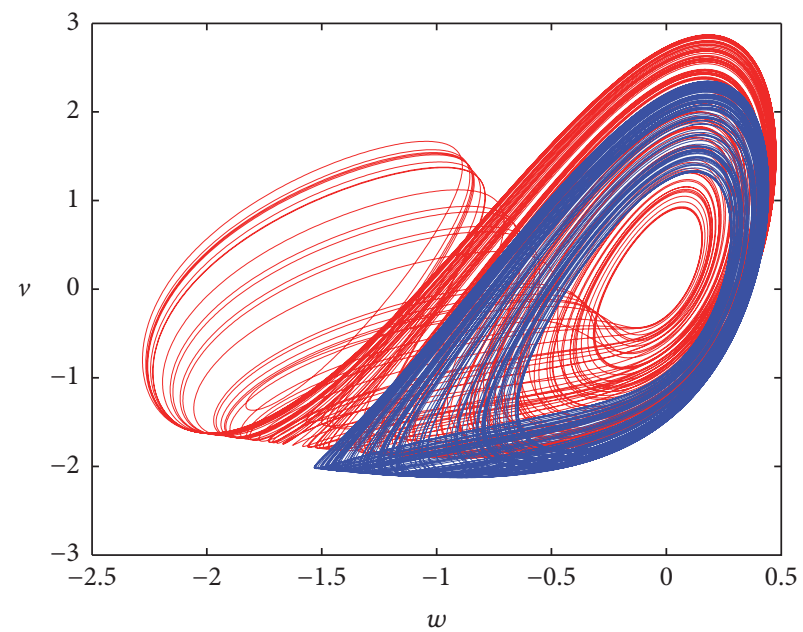

(f)

FIGURE 15: Coexisting attractors with the same parameters but different initial values. (a) Basin of attraction versus $x(0)$ and $y(0)$. (b) Coexisting limit cycle and point attractor. (c) Two coexisting limit cycles. (d) Coexisting chaotic attractor and point attractor. (e) Coexisting chaotic attractor and limit cycle. (f) Two coexisting chaotic attractors. 
state, corresponding to a chaotic attractor. Figure 15 shows the variations of the attractors versus initial values $x(0)$ and $y(0)$. Figure $15(\mathrm{a})$ is the basin of attraction, indicating that the different attractors appear under the changes of the two initial values at the same time. When $x(0)$ is in the range $[-0.02,0.024]$ and $y(0)$ is in the range $[0.1,0.4]$, the system exhibits different limiting cycles (as shown in the orange region and green region of Figure 15(a)) and chaotic attractors (as shown in the blue and red regions of Figure 15(a)).

When initial condition is set by point in the orange region $\mathrm{O}$, for example, let initial condition $(x(0), y(0))=$ $(0.02,0.4)$ (other initial conditions: $(z(0), w(0), v(0))=$ $(0.01,0.01,0.01))$, the system is attracted in a periodic orbit colored orange in Figure 15(b); but when $(x(0), y(0))=$ $(0.3,0.1)$ and other initial conditions remain unchanged, the system has a point attractor shown by a black point.

When initial condition is set by the point in the green region $\mathrm{G}$, the system has another kind of limiting cycle, which is shown as green cycle in Figure 15(c), where the initial condition is $(0,02,0.25,0.01,0.01,0.01)$. Figure $15(\mathrm{~d})$ shows the chaotic attractor and point attractor, where the blue orbit represents the chaotic attractor for initial condition of $(-0.02,0.1,0.01,0.01,0.01)$. Figure $15(\mathrm{e})$ represents the chaotic attractor (red) and limiting cycle (green) belonging to initial conditions of $(0.024,0.1,0.01$, $0.01,0.01)$ and $(0.02,0.3,0.01,0.01,0.01)$, respectively. Finally Figure 15(f) shows two different chaotic orbits with two different initial values $(-0.02,0.1,0.01,0.01,0.01)$ (blue) and $(0.024,0.1,0.01,0.01,0.01)$ (red).

\section{DSP Experiment}

The digitization of chaotic system can generate PN sequence, which can be used in secure communications. Based on DSP's powerful data processing capability and high speed, it is a good choice to achieve high precision and high efficient iterative algorithm of a complex chaotic system by using DSP technology. DSP hardware system is using ICETEK-VC5502-AE evaluation board with the CCStudiov3.3 software platform. In order to digitize the chaotic system with memristor and memcapacitor, continuous differential equation (12) is needed to be discretized using Euler method [25]. The Euler method is mainly based on the definition of the derivative, which is defined as

$$
f^{\prime}(x)=\lim _{\Delta t \rightarrow 0} \frac{x\left(t_{n}+\Delta t\right)-x\left(t_{n}\right)}{\Delta t}=\lim _{\Delta t \rightarrow 0} \frac{x_{n+1}-x_{n}}{\Delta t} .
$$

When $\Delta t$ tends $0,(20)$ can be approximated as

$$
f^{\prime}(x) \approx \frac{x_{n+1}-x_{n}}{\Delta t}=\frac{x(n+1)-x(n)}{\Delta t} .
$$

According to (21), the chaotic system can be discretized as

$$
\begin{aligned}
x_{p}= & x_{p-1} \\
& +\tau n\left(j\left(\left(c-d w_{p-1}\right) z_{p-1}-x_{p-1}\right)-y_{p-1}\right) \\
y_{p}= & y_{p-1}+\tau m\left(x_{p-1}-\left(a-b v_{p-1}\right) y_{p-1}\right) \\
z_{p}= & z_{p-1}+\tau k\left(c-d w_{p-1}\right) z_{p-1} \\
& +j\left(x_{p-1}-\left(c-d w_{p-1}\right) z_{p-1}\right) \\
w_{p}= & w_{p-1}+\tau z_{p-1}, \\
v_{p}= & v_{p-1}+\tau y_{p-1},
\end{aligned}
$$

where $a=0.01, b=0.1, c=0.7, d=-2, n=0.17, j=4.8$, $m=7.35$, and $k=2.1$; initial condition is $(0.01,0.1,0.01$, $0.01,0.01$ ); and quantization precision is $\tau=0.01$. A discrete sequence can be obtained by iterating (22).

There are many quantization methods of chaotic sequences. For simplicity, the threshold method $[25,26]$ is adopted in this paper. Let the chaotic mapping be

$$
f(p)=x_{p} \quad p=1,2,3, \ldots
$$

The digitized chaotic sequence is set as $u_{p}$, and the quantization method is described as

$$
u_{p}= \begin{cases}1, & \text { if } x_{p} \geq q \\ 0, & \text { else }\end{cases}
$$

where $q$ denotes the quantization threshold.

According to (22) and (24), we can write the discrete and digitized programs in the CCS environment and then download the prepared programs to DSP evaluation board. The experimental binary sequences observed by an oscilloscope are shown in Figure 16(a). The binary sequences can be transforming the continuous chaotic waveforms or attractors by a D/A convertor, which is shown in Figures 16(b)-16(f).

\section{PN Sequence and Its NIST Test}

According to the proposed chaotic system, digital pseudorandom sequences can be generated by using the DSP technology. In order to understand the random characteristics of the sequences, the test software package STS [27], which was introduced by National Institute of Standard and Technology (NIST) of America, was used to test the generated sequences.

The NIST test suit, the most authoritative tool for pseudorandom testing currently, is a statistical software package consisting of 15 tests, which is developed to test the 757 randomness of binary sequences produced by pseudorandom signal generators. The 2.0 version of the test suite package is used in the experiment.

NIST provides two criteria for evaluating the performance of a sequence, that is, passing rate of the sequences 


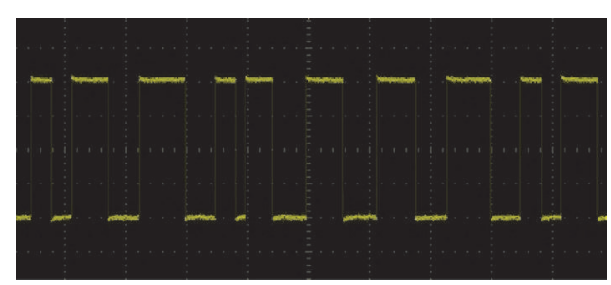

(a)

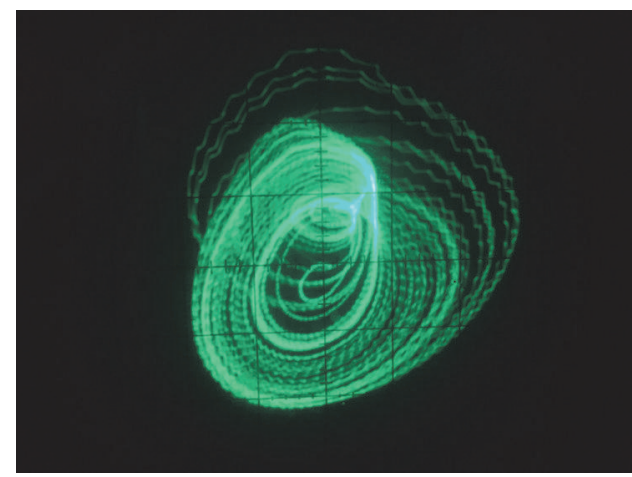

(c)

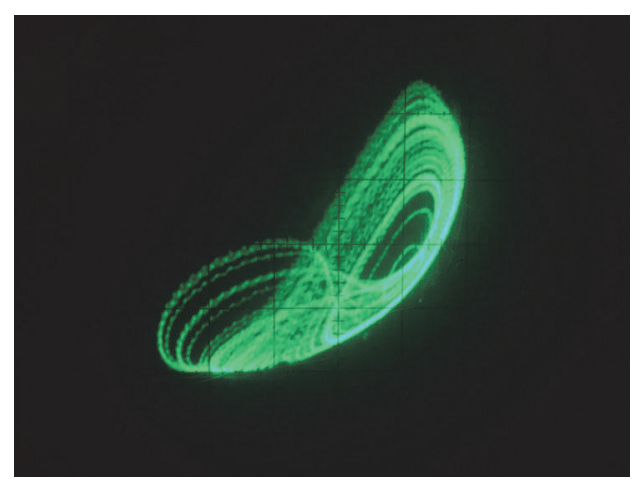

(e)

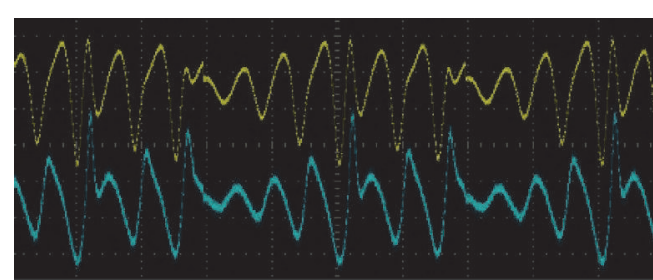

(b)

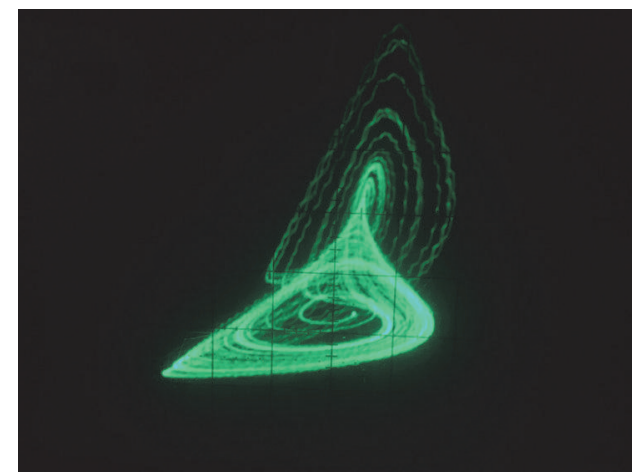

(d)

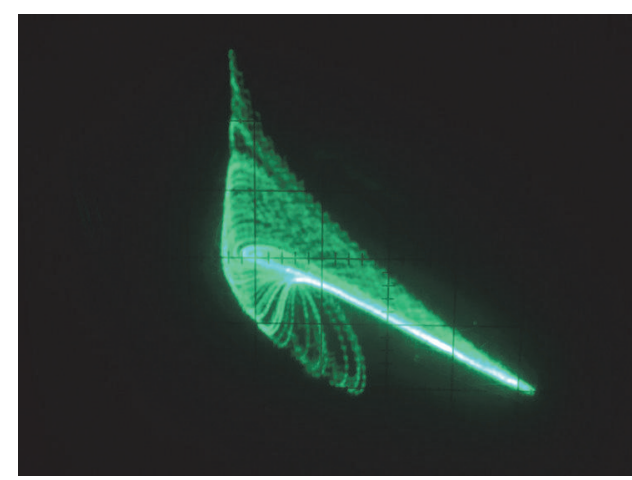

(f)

FIGURE 16: Experimental results observed from oscillator. (a) Binary sequence. (b) Continuous waveforms. (c) Phase diagram of $x-y$. (d) Phase diagram of $y-z$. (e) Phase diagram of $w-v$. (f) Phase diagram of $v-x$.

and uniform distribution of $P$ values. A final analysis report is obtained from the test suite package with relevant tested values, including test statistics (proportion) and $P$ value for each test.

Now, we evaluate the randomness of the proposed system by testing its binary sequences and using the NIST test suit and compare the randomness with that of the well-known Lorenz system.

For a generated binary sequence of a given length $n$, we divide the sequence into $m$ nonoverlapping parts with the same length $k(m=n / k)$, where $n=1,000,000,000$ and $m=$ 1000 , so $k=1,000,000$.

The NIST test results of the proposed system are shown in Table 1. For comparison, Table 2 shows the NIST test results of the well-known Lorenz system, and Figure 17 shows the histograms of $P$ value and passing rate simultaneously. From Tables 1 and 2 and Figure 17, we can conclude that the stochastic performances of the proposed chaotic oscillator are better than those of the Lorenz system.

\section{Conclusion}

In this paper, we design a chaotic oscillator for the prestudy of the new PN generator using the hybrid oscillator circuit containing memristor and memcapacitor as a seed. The results of theory analysis and experiment show that this generator can exhibit some complex dynamical characteristics, including chaos, hyperchaos, complex bifurcation, and coexisting oscillation. Furthermore, digital circuit experiment of the oscillator is performed by a DSP evaluation board, for verifying realization of the system. Random properties of the PN sequences generated from the system fully meet the NIST standards and are better than those of the wellknown Lorenz system. Therefore, the proposed memristor and memcapacitor based oscillator can be used for designing $\mathrm{PN}$ sequence generators as a new random signal seed, which has good random properties and the potential application value in the field of secret communication and chaotic cryptography. 


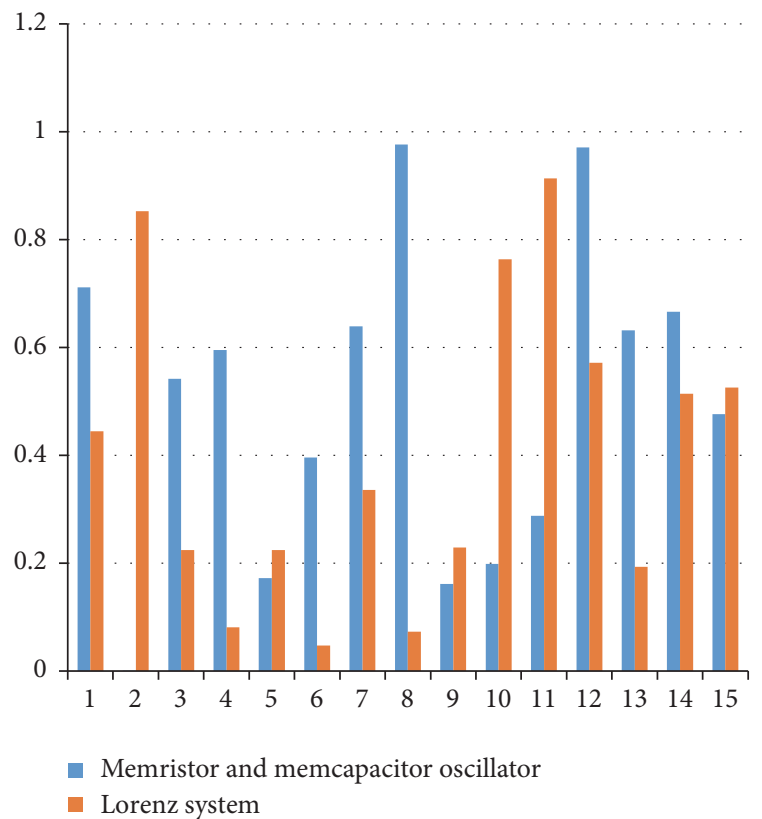

(a)

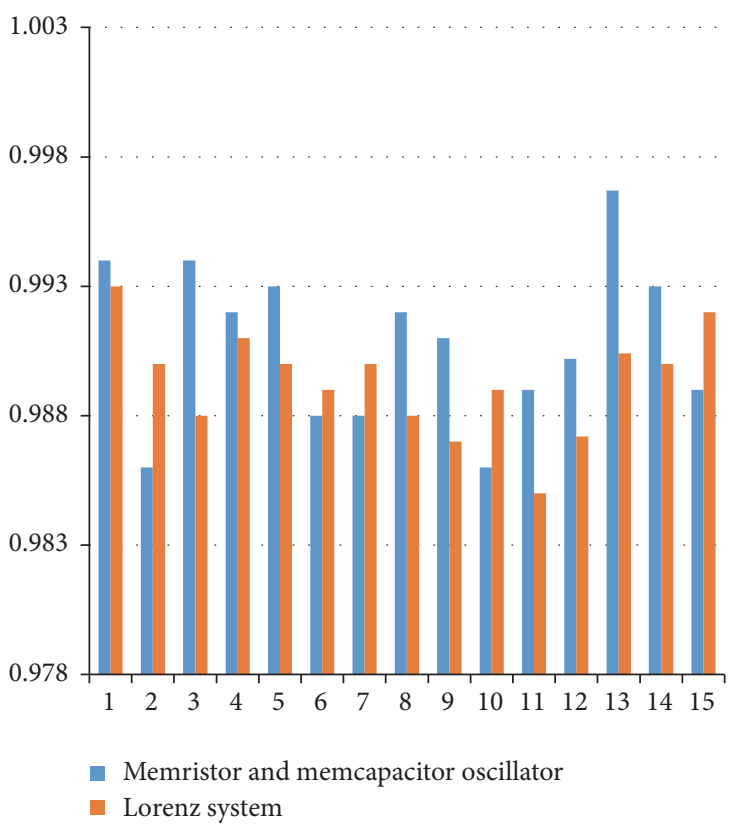

(b)

FIGURE 17: Random performance comparison between the memristor and memcapacitor based chaotic system and the Lorenz system. (a) $P$ value. (b) Passing rate.

TABLE 1: NIST test results of the proposed chaotic system.

\begin{tabular}{lcc}
\hline Statistical test & $P$-value & Proportion \\
\hline Frequency & 0.711601 & 0.9940 \\
Block frequency & 0.000363 & 0.9860 \\
Cumulative sums & 0.542228 & 0.9940 \\
Runs & 0.595549 & 0.9920 \\
Longest run & 0.172816 & 0.9930 \\
Rank & 0.395940 & 0.9880 \\
Fft & 0.639202 & 0.9880 \\
Nonoverlapping template & 0.976878 & 0.9920 \\
Overlapping template & 0.161703 & 0.9910 \\
Universal & 0.199045 & 0.9860 \\
Approximate entropy & 0.288249 & 0.9890 \\
Random excursions & 0.971017 & 0.9902 \\
Random excursions variant & 0.631999 & 0.9967 \\
Serial & 0.666245 & 0.9930 \\
Linear complexity & 0.476911 & 0.9890 \\
\hline
\end{tabular}

\section{Competing Interests}

The authors declare that there is no conflict of interests regarding the publication of this paper.

\section{Acknowledgments}

This work was supported by the National Natural Science Foundation of China under Grant nos. 61271064 and
TABLE 2: NIST test results of Lorenz chaotic system.

\begin{tabular}{lcc}
\hline Statistical test & $P$-value & Proportion \\
\hline Frequency & 0.444691 & 0.9930 \\
Block frequency & 0.853049 & 0.9900 \\
Cumulative sums & 0.224821 & 0.9880 \\
Runs & 0.08151 & 0.9910 \\
Longest run & 0.224821 & 0.9900 \\
Rank & 0.047785 & 0.9890 \\
Fft & 0.336111 & 0.9900 \\
Nonoverlapping template & 0.073417 & 0.9880 \\
Overlapping template & 0.229559 & 0.9870 \\
Universal & 0.763677 & 0.9890 \\
Approximate entropy & 0.914025 & 0.9850 \\
Random excursions & 0.571919 & 0.9872 \\
Random excursions variant & 0.193194 & 0.9904 \\
Serial & 0.514124 & 0.9900 \\
Linear complexity & 0.526105 & 0.9920 \\
\hline
\end{tabular}

60971046 and the Program for Zhejiang Leading Team of S\&T Innovation under Grant no. 2010R50010.

\section{References}

[1] L. O. Chua, "Memristor-the missing circuit element," IEEE Transactions on Circuit Theory, vol. 18, no. 5, pp. 507-519, 1971.

[2] D. B. Strukov, G. S. Snider, D. R. Stewart, and R. S. Williams, "The missing memristor found," Nature, vol. 453, pp. 80-83, 2008 . 
[3] L. O. Chua, "Nonlinear circuit theory," in Proceedings of the Guest Lectures of the European Conference on Circuit Theory and Design, vol. 81, 1978.

[4] L. O. Chua, "Nonlinear circuit foundations for nano devices," Proceedings of the IEEE, vol. 91, no. 11, pp. 1830-1859, 2003.

[5] M. Di Ventra, Y. V. Pershin, and L. O. Chua, "Circuit elements with memory: memristors, memcapacitors, and meminductors," Proceedings of the IEEE, vol. 97, no. 10, pp. 1717-1724, 2009.

[6] B. C. Bao, P. Jiang, H. G. Wu, and F. W. Hu, "Complex transient dynamics in periodically forced memristive Chua's circuit," Nonlinear Dynamics, vol. 79, no. 4, pp. 2333-2343, 2015.

[7] M. Itoh and L. O. Chua, "Memristor oscillators," International Journal of Bifurcation and Chaos, vol. 18, no. 11, pp. 3183-3206, 2008.

[8] B. Muthuswamy and L. O. Chua, "Simplest chaotic circuit," International Journal of Bifurcation and Chaos, vol. 20, no. 5, pp. 1567-1580, 2010.

[9] A. Buscarino, L. Fortuna, M. Frasca, and L. V. Gambuzza, "A chaotic circuit based on Hewlett-Packard memristor," Chaos, vol. 22, no. 2, Article ID 023136, 2012.

[10] D. S. Yu, Y. Liang, H. Chen, and H. H. C. Iu, "Design of a practical memcapacitor emulator without grounded restriction," IEEE Transactions on Circuits and Systems II: Express Briefs, vol. 60, no. 4, pp. 207-211, 2013.

[11] A. L. Fitch, H. H. C. Iu, and D. S. Yu, "Chaos in a memcapacitor based circuit," in Proceedings of the IEEE International Symposium on Circuits and Systems (ISCAS '14), pp. 482-485, Melbourne, Australia, June 2014.

[12] D. Biolek and V. Biolkova, "Mutator for transforming memristor into memcapacitor," Electronics Letters, vol. 46, no. 21, pp. 14281429, 2010.

[13] X. Y. Wang, A. L. Fitch, H. H. C. Iu, and W. G. Qi, "Design of a memcapacitor emulator based on a memristor," Physics Letters A, vol. 376, no. 4, pp. 394-399, 2012.

[14] M. E. Fouda and A. G. Radwan, "Charge controlled memristorless memcapacitor emulator," Electronics Letters, vol. 48, no. 23, pp. 1454-1455, 2012.

[15] D. Biolek, Z. Biolek, and V. Biolkova, "SPICE modelling of memcapacitor," Electronics Letters, vol. 46, no. 7, pp. 520-522, 2010.

[16] D. Biolek, Z. Biolek, and V. Biolková, "Behavioral modeling of memcapacitor," Radioengineering, vol. 20, no. 1, pp. 228-233, 2011.

[17] G.-Y. Wang, B.-Z. Cai, P.-P. Jin, and T.-L. Hu, "Memcapacitor model and its application in a chaotic oscillator," Chinese Physics B, vol. 25, no. 1, Article ID 010503, pp. 489-500, 2015.

[18] M. E. Fouda and A. G. Radwan, "Resistive-less memcapacitorbased relaxation oscillator," International Journal of Circuit Theory and Applications, vol. 43, no. 7, pp. 959-965, 2015.

[19] J. Kengne, Z. N. Tabekoueng, V. K. Tamba, and A. N. Negou, "Periodicity, chaos, and multiple attractors in a memristorbased Shinriki's circuit," Chaos, vol. 25, no. 10, Article ID 103126, 2015.

[20] F. Yuan, G. Y. Wang, Y. R. Shen et al., "Coexisting attractors in a memcapacitor-based chaotic oscillator," Nonlinear Dynamics, vol. 85, no. 1, pp. 37-50, 2016.

[21] M. Chen, M. Li, Q. Yu, B. Bao, Q. Xu, and J. Wang, "Dynamics of self-excited attractors and hidden attractors in generalized memristor-based Chua's circuit," Nonlinear Dynamics, vol. 81, no. 1-2, pp. 215-226, 2015.
[22] F. Yuan, G. Wang, and X. Wang, "Extreme multistability in a memristor-based multi-scroll hyper-chaotic system," Chaos. An Interdisciplinary Journal of Nonlinear Science, vol. 26, no. 7, Article ID 073107, 2016.

[23] Y. V. Pershin and M. Di Ventra, "Emulation of floating memcapacitors and meminductors using current conveyors," Electronics Letters, vol. 47, no. 4, pp. 243-244, 2011.

[24] A. L. Fitch, H. H. C. Iu, and D. S. Yu, "Chaos in a memcapacitor based circuit," in Proceedings of the IEEE International Symposium on Circuits and Systems (ISCAS '14), pp. 482-485, IEEE, Melbourne, Australia, June 2014.

[25] T. Kohda and A. Tsuneda, "Statistics of chaotic binary sequences," IEEE Transactions on Information Theory, vol. 43, no. 1, pp. 104-112, 1997.

[26] M. E. Yalçın, J. A. K. Suykens, and J. Vandewalle, "True random bit generation from a double-scroll attractor," IEEE Transactions on Circuits and Systems I: Regular Papers, vol. 51, no. 7, pp. 13951404, 2004.

[27] A. Rllkhin, J. Soto, and J. Nechvatal, "A Statistical Test Suite for Random and Pseudorandom Number-Generators for Cryptographic Applications [EB/OL]," https://ulib.aub.edu.lb/FEBK/ febk000279.pdf. 


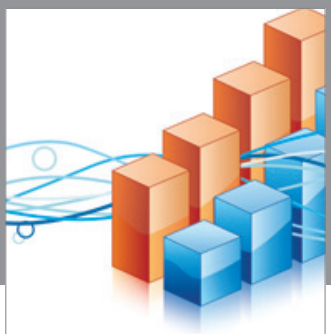

Advances in

Operations Research

vatem alat4

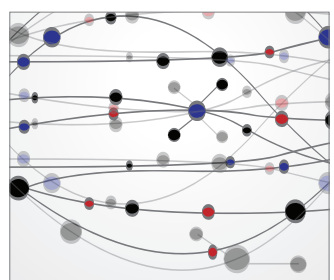

\section{The Scientific} World Journal
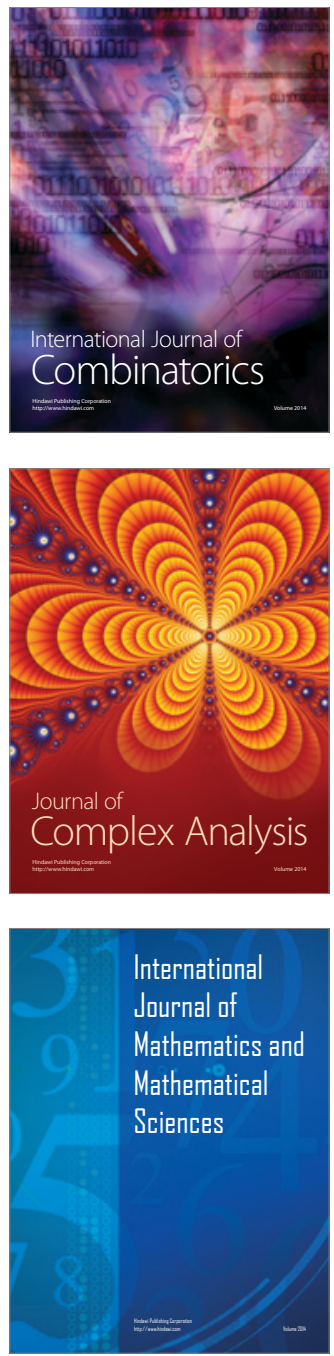
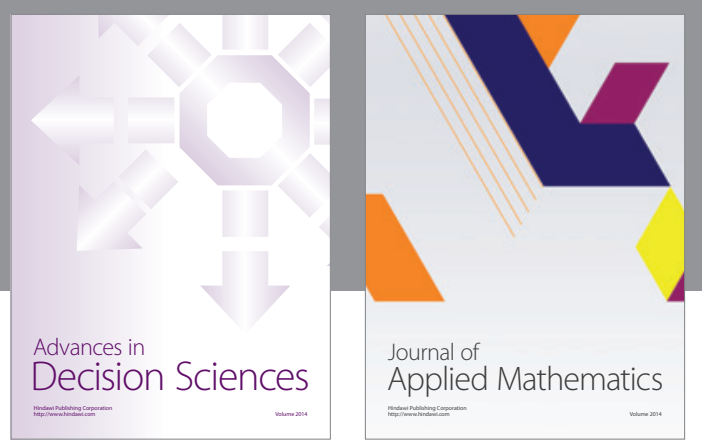

Algebra

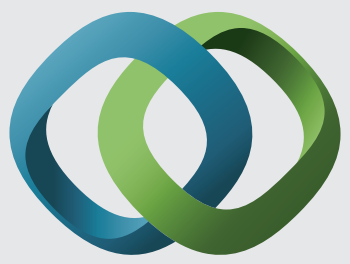

\section{Hindawi}

Submit your manuscripts at

http://www.hindawi.com
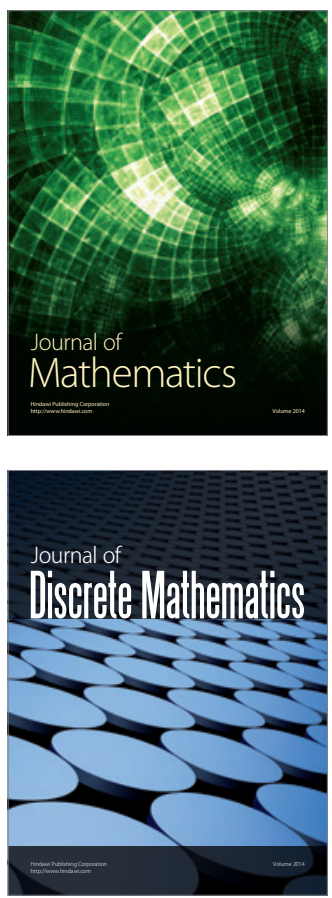

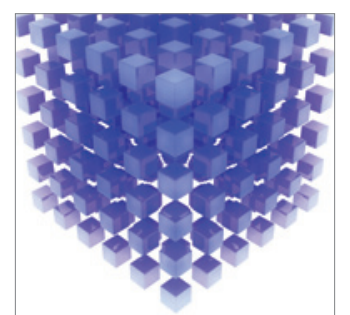

Mathematical Problems in Engineering
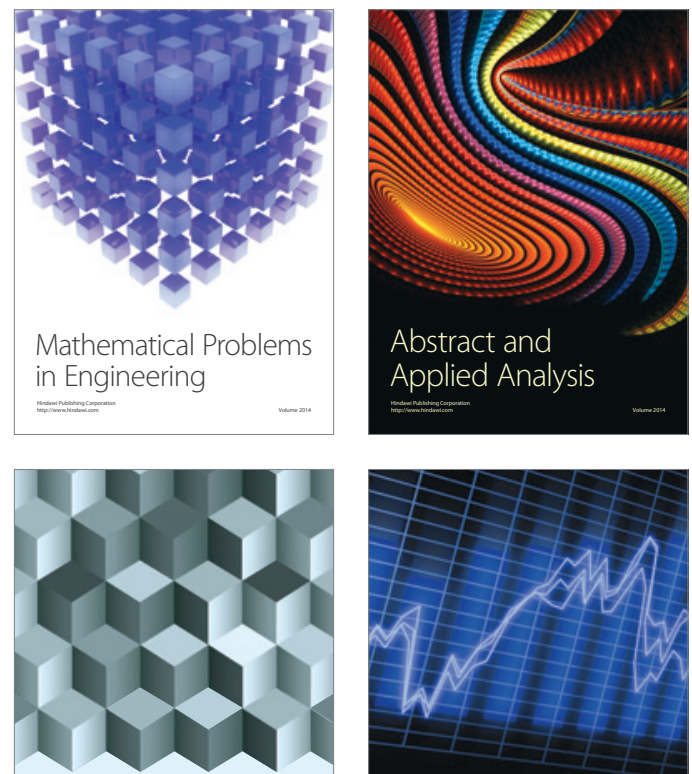

Journal of

Function Spaces

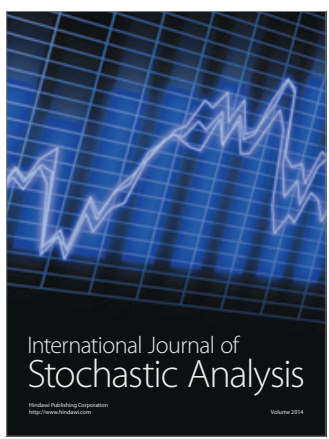

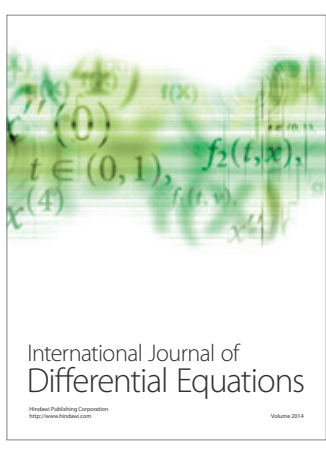
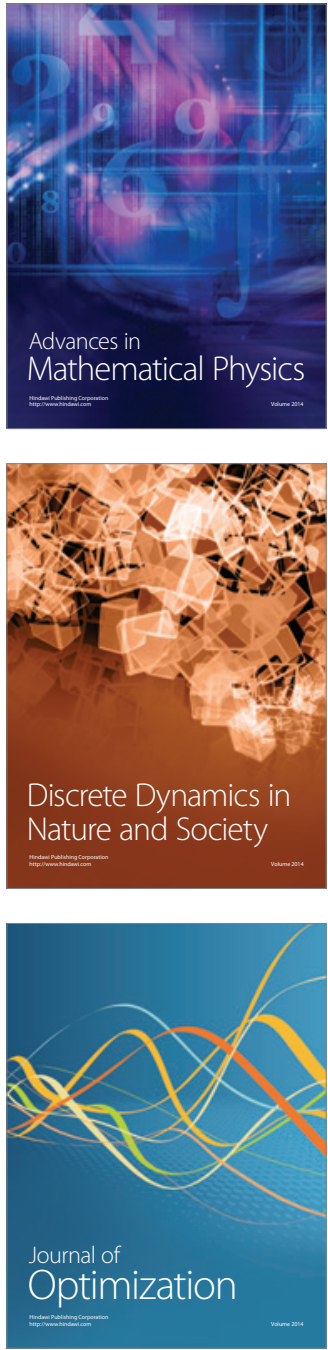